\title{
ON CERTAIN FAMILIES OF ORBITS WITH ARBITRARY MASSES IN THE PROBLEM OF THREE BODIES*
}

BY

\author{
F. H. MURRAY
}

This paper is devoted to the study of certain families of orbits in the problem of three bodies, which lie in the neighborhood of known orbits of simple types, but in which the masses are arbitrary and fixed. The classical method of variation of a parameter which enters into the equations of motion cannot, therefore, be employed; instead, extensive use is made of methods in which the notion of invariant relation $\dagger$ plays a prominent rôle.

In the first two paragraphs are developed certain implications regarding the equations of variation, which result from the existence of a set of invariant relations satisfied by the generating solution of a differential system, following which certain properties of isosceles triangle solutions with axis of symmetry in the problem of three bodies are established. The latter part of the paper is given up to the study of plane orbits which lie in the neighborhood of the straight line solutions, or in the neighborhood of the equilateral triangle solutions, respectively ; in this study a simple reduction of the equations of motion in the plane is obtained, which is found especially convenient.

Many of the results obtained concerning these last solutions have been given by $\mathrm{D}$. Buchanan, $\ddagger$ but the method employed here has made it possible to go farther in certain respects. Certain related questions of stability will be taken up in a later paper.

\section{INVARIANT RELATIONS}

Suppose given a system of differential equations

$$
\frac{d x_{i}}{d t}=X_{i}\left(x_{1}, x_{2}, \cdots, x_{n}\right) \quad(i=1, \cdots, n)
$$

* Presented to the Society, September 7, 1923; received by editors in January, 1924.

† See Poincaré, Les Méthodes Nouvelles de la Mécanique Celeste, vol. 1, p. 45.

$\ddagger$ Rendicontidel Circolo Matematico di Palermo, vol. 25 (1921), pp. 1-25; these, Transactions, vol. 23 (1922), pp. 409-431. 
in which $X_{i}$ is analytic in its arguments, if $\left(x_{1}, \cdots, x_{n}\right)$ lies within a region $D$. Suppose the equations

$$
\begin{gathered}
\varphi_{1}\left(x_{1}, \ldots, x_{n}\right)=0, \\
\ldots \ldots \\
\varphi_{r}\left(x_{1}, \ldots, x_{n}\right)=0
\end{gathered}
$$

to form a set of invariant relations for solutions of (1). If a particular solution of (1) is defined by the equations

$$
x_{1}=w_{1}(t), \cdots, x_{n}=w_{n}(t)
$$

in an interval $\delta:(0 \leqq t \leqq T)$, let $R$ be the region composed of all points $\left(x_{1}, \cdots, x_{n}\right)$ of $D$ which satisfy the inequalities

(a)

$$
\left|x_{i}-w_{i}(t)\right|<a \quad(i=1, \cdots, n),
$$

for some $t$ on $\delta$. It will be assumed that $\varphi_{1}, \cdots, \varphi_{\mathrm{r}}$ are analytic within $R$, and that for every value of $t$ on $\delta$ some $r$-rowed determinant of the matrix

$$
\left\|\begin{array}{cccc}
\frac{\partial \varphi_{1}}{\partial x_{1}} & \frac{\partial \varphi_{1}}{\partial x_{2}} & \cdots & \frac{\partial \varphi_{1}}{\partial x_{n}} \\
\frac{\partial \varphi_{2}}{\partial x_{1}} & \frac{\partial \varphi_{2}}{\partial x_{2}} & \cdots & \frac{\partial \varphi_{2}}{\partial x_{n}} \\
\cdots & \cdots & \cdots \\
\frac{\partial \varphi_{r}}{\partial x_{1}} & \frac{\partial \varphi_{r}}{\partial x_{2}} & \cdots & \frac{\partial \varphi_{r}}{\partial x_{n}}
\end{array}\right\|
$$

is different from zero in the region defined by inequalities $(a)$.

Under these hypotheses every value $t^{0}$ of $t$ on $\delta$ lies in an interval $\delta^{\prime}:\left|t-t^{0}\right|<\eta$ such that the equations

$$
\begin{gathered}
y_{1}=\varphi_{1}\left(x_{1}, \ldots, x_{n}\right), \\
\cdots \cdots \\
y_{r}=\varphi_{r}\left(x_{1}, \ldots ., x_{n}\right)
\end{gathered}
$$

can be solved for $r$ of the variables $x_{1}, \cdots, x_{n}$; if these are $x_{1}, \cdots, x_{r}$,

$$
x_{i}=\psi_{i}\left(y_{1}, \cdots, y_{r}, x_{r+1}, \cdots, x_{n}\right) \quad(i=1, \cdots, r)
$$

in a region

( $\beta$ )

$$
\left|y_{i}\right|<a^{\prime}, \quad\left|x_{r+k}-w_{r+k}(t)\right|<a^{\prime},
$$


and inequalities $(\beta)$ have $(a)$ as a consequence if $a^{\prime}$ is suitably chosen, ${ }^{*}$ $t$ on $\delta^{\prime}$. Also, $\psi_{i}$ is analytic in its arguments in the region defined by $(\beta)$, if $t$ is on $\delta^{\prime}$. If $x_{r+1}=y_{r+1}, \cdots, x_{n}=y_{n}$,

$$
\begin{array}{ll}
\frac{d y_{i}}{d t}=\sum_{k=1}^{n} X_{k} \frac{\partial \varphi_{i}}{\partial x_{k}}=Y_{i}\left(y_{1}, \cdots, y_{n}\right) & (i=1, \cdots, r), \\
\frac{d y_{l}}{d t}=X_{l}\left(x_{1}, \cdots, x_{n}\right)=Y_{l}\left(y_{1}, \cdots, y_{n}\right) & (l=r+1, \cdots, n) .
\end{array}
$$

The functions $Y_{i}$ are analytic in their arguments, and may be expanded as power series in $y_{1}, \cdots, y_{r}$ :

$$
Y_{i}=\sum A_{a_{1} \cdots \alpha_{r}}^{(i)} y_{1}^{a_{1}} \cdots y_{r}^{a_{r}},
$$

$$
\left|y_{i}\right|<a^{\prime \prime}, \quad\left|x_{k}-w_{k}(t)\right|<a^{\prime \prime} \quad(i=1, \cdots, r ; \quad k=r+1, \cdots, n) .
$$

Since the equations (2) form a set of invariant relations, $Y_{1}, \cdots, Y_{r}$ must vanish when $y_{1}=y_{2}=\cdots=y_{r}=0$. Hence

$$
A_{0 \ldots 0}^{(i)}=0 \text {, }
$$

$$
\frac{d y_{i}}{d t}=a_{i 1} y_{1}+a_{i 2} y_{2}+\cdots+a_{i r} y_{r}+\psi_{2}^{(i)} \quad(i=1, \cdots, r)
$$

where $\psi_{2}^{(1)}$ is of at least the second degree with respect to $y_{1}, \cdots, y_{r}$. In terms of the original variables

$$
\frac{d \varphi_{i}}{d t}=\sum_{k=1}^{r} A_{i k} \varphi_{k}+\psi_{2}^{(i)}\left(\varphi_{1}, \cdots, \varphi_{r}, x_{r+1}, \cdots, x_{n}\right),
$$

* For, from the classical theorems on implicit functions it follows that corresponding to each value $t^{0}$ of $t$ on $\delta$, there exists a region

$\left(\delta_{1}\right) \quad\left|y_{i}\right|<b, \quad\left|x_{k}-w_{k}\left(t^{0}\right)\right|<b$

$$
(i=1, \cdots, r ; k=r+1, \cdots, n)
$$

for some $r$ variables $x_{1}, \cdots, x_{r}$, such that the functions

$$
x_{i}=\Psi_{i}\left(y_{1}, \cdots, y_{r}, x_{r+1}, \cdots, x_{n}\right) \quad(i=1, \cdots, r)
$$

are defined within $\left(\delta_{1}\right)$ and inequalities $(a)$ are a consequence of $\left(\delta_{1}\right)$. Suppose

Then if $\left|t-t_{0}\right|<b / 2 M$, from (1),

$$
a^{\prime}=b / 2, \quad\left|X_{i}\right|<M \quad(i=r+1, \cdots, n) .
$$

Hence if

$$
\left|w_{k}(t)-w_{k}\left(t^{0}\right)\right|<a^{\prime} \quad(k=r+1, \cdots, n) .
$$

(B) $\quad\left|y_{i}\right|<a^{\prime},\left|x_{k}-w_{k}(t)\right|<a^{\prime},\left|t-t_{0}\right|<a^{\prime} / M \quad(i=1, \cdots, r ; k=r+1, \cdots, n)$, it follows that $\left|x_{k}-w_{k}\left(t^{\circ}\right)\right|<b$, and inequalities $\left(\delta_{1}\right)$ are a consequence of $(\beta)$. 
and the series on the right must converge if $\left(x_{1}, \cdots, x_{n}\right)$ lies within a region

$$
\left|x_{i}-w_{i}(t)\right|<\bar{a}^{\prime \prime}, \quad\left|t-t^{0}\right|<\eta \quad(i=1, \cdots, n),
$$

which corresponds to that defined by inequalities $\left(\beta^{\prime}\right)$.

Since every $t$ on $\delta$ lies in an interval $\delta^{\prime}$ for which equations of the form $\left(2^{\prime \prime}\right)$ hold, it follows from the Heine-Borel theorem that $\delta$ can be covered by a finite number of intervals of this form, and these can be chosen as overlapping intervals $\delta^{\prime}$; if $\bar{a}$ is the smallest of the quantities $\bar{a}^{\prime \prime}$, inequalities $\left(a^{\prime}\right)$ are a consequence of inequalities

$$
\left|x_{i}-w_{i}(t)\right|<\bar{a}, 0 \leqq t \leqq T .
$$

Since the coefficients of the series on the right of $\left(2^{\prime \prime}\right)$, defined for a region $\delta^{\prime}$, must coincide with the corresponding ones defined for $\delta^{\prime \prime}$ in the region common to $\delta^{\prime}$ and $\delta^{\prime \prime}$, each must be the analytic prolongation of the other. It follows that equations $\left(2^{\prime \prime}\right)$ are valid under the hypotheses concerning $X_{i}, \varphi_{k}$, within the region defined by inequalities $(\bar{a})$.

Now if

$$
X(f)=\sum_{i=1}^{n} X_{i} \frac{\partial f}{\partial x_{i}},
$$

then if $f\left(x_{1}, \cdots, x_{n}\right)$ is defined as a function of $t$ by means of equations (1),

$$
f(t)=f\left(x_{1}^{0}, \cdots \cdot x_{n}^{0}\right)+t X(f)+\frac{t^{2}}{2 !} X[X(f)]+\cdots \cdot
$$

If $\varphi_{i}\left(x_{1}, \cdots, x_{n}\right)$ is expanded in this manner, and if $\varphi_{i}\left(x_{1}^{0}, \cdots, x_{n}^{0}\right)=0$ $(i=1, \cdots, r)$, from $\left(2^{\prime \prime}\right) X\left(\varphi_{i}\right)=0, X\left[X\left(\varphi_{i}\right)\right]=0, \cdots$ and hence $\varphi_{i}=0$, for all values of $t$ on $\delta$. This result, announced by Poincaré (loc. cit.), seems of sufficient importance to warrant the detailed demonstration given above.

If the change of variables

$$
y_{1}=\varphi_{1}, \cdots, y_{r}=\varphi_{r}, y_{r+1}=y_{r+1}^{0}+z_{r+1}, \cdots, y_{n}=y_{n}^{0}+z_{n}
$$

is made, where $y_{r+1}^{0}, \cdots, y_{n}^{0}$ are a solution of the system

$$
\frac{d y_{i}^{0}}{d t}=Y_{i}\left(0, \cdots, 0, y_{r+1}^{0}, \cdots, y_{n}^{0}\right) \quad(i=r+1, \cdots, n) \text {, }
$$

we obtain

$$
\frac{d z_{k}}{d t}=\sum_{0=1}^{r} \frac{\partial Y_{k}}{\partial y_{0}} y_{0}+\sum_{0=r+1}^{n} \frac{\partial Y_{k}}{\partial y_{0}} z_{c}+\psi_{2}^{(k)}\left(y_{1}, \cdots, y_{r}, z_{r+1}, \cdots, z_{n}\right) .
$$


The equations of variation of the system $\left(1^{\prime}\right)$ are therefore in the form
(a) $\frac{d \bar{y}_{i}}{d t}=a_{i 1} \bar{y}_{1}+a_{i 2} \bar{y}_{2}+\cdots \cdot+a_{i r} \bar{y}_{r}$
$(i=1, \cdots \cdot r)$,
(b) $\frac{d \bar{z}_{k}}{d t}=b_{k 1} \bar{y}_{1}+\cdots \cdot+b_{k r} \bar{y}_{r}+b_{k, r+1} \bar{z}_{r+1}+\cdots+b_{k n} \bar{z}_{n}$

$$
(k=r+1, \cdots \cdot n) \text {. }
$$

If the system (a) is integrated first, (b) can be integrated as a nonhomogeneous system in $\bar{z}_{r+1}, \cdots, \bar{z}_{n}$.

The system obtained from $\left(1^{\prime}\right)$ by giving $y_{1}, \cdots, y_{r}$ the value zero will be called the reduced system with respect to the relations (2). This reduced system may admit another set of invariant relations, by means of which a second reduced system is obtained, etc. In this manner any given set of invariant relations can be grouped into sub-sets,

$$
\begin{array}{ll}
S_{1}: & \left(\varphi_{1}^{\prime}, \cdots \cdot, \varphi_{r_{1}}^{\prime}\right), \\
S_{2}: & \left(S_{1}, \varphi_{1}^{\prime \prime}, \cdot \cdot, \varphi_{r_{2}}^{\prime \prime}\right), \\
& \cdot \cdot \cdot \cdot \cdot \cdot \cdot \\
S_{m}: & \left(S_{m-1}, \varphi_{1}^{(m)} \cdot \cdots \cdot, \varphi_{r_{m}}^{(m)}\right),
\end{array}
$$

such that when the functions of the set $S_{k-1}$ are set equal to zero, the relations

$$
\varphi_{1}^{(k)}=0, \cdots, \varphi_{r_{k}}^{(k)}=0
$$

form a set of invariant relations. This division into sub-sets can in general be made in a variety of ways.

It is seen immediately that a corresponding grouping of the equations of (a) can be made; these are necessarily in the form (if $s_{k}=r_{1}+r_{2}+\cdots+r_{k}$ )

$$
\begin{aligned}
& \left(S_{1}{ }^{\prime}\right) \quad \frac{d \bar{y}_{i}^{\prime}}{d t}=\sum_{k=1}^{r_{1}} a_{i k} \bar{y}_{k}{ }^{\prime} \\
& \left(i=1, \cdot \cdot, r_{1}\right) \text {, } \\
& \left(S_{2}{ }^{\prime}\right) \frac{d \bar{y}_{i}{ }^{\prime \prime}}{d t}=\sum_{k=1}^{r_{1}} a_{s_{1}+i, k} \bar{y}_{k}{ }^{\prime}+\sum_{k=1}^{r_{2}} a_{\theta_{1}+i, s_{1}+k} \bar{y}_{k}^{\prime \prime} \\
& \left(i=1, \cdot \cdot, r_{2}\right), \\
& \left(S_{m}{ }^{\prime}\right) \frac{d \bar{y}_{i}^{(m)}}{d t}=\sum_{k=1}^{r_{1}} a_{e_{m-1+i, k}} \bar{y}_{k}^{\prime}+\sum_{k=1}^{r_{2}} a_{e_{m-1+i, e_{1}+k}} \bar{y}_{k}^{\prime \prime}+\cdots \\
& +\sum_{k=1}^{r_{m}} a_{s_{m-1}+i, s_{m-1}+k} \bar{y}_{k}^{(m)} \quad\left(i=1,2, \cdots, r_{m}\right) .
\end{aligned}
$$


The integration of equations $(a)$ is therefore reduced to the successive integrations of the sets $\left(S_{1}{ }^{\prime}\right),\left(S_{2}{ }^{\prime}\right), \cdots,\left(S_{m}{ }^{\prime}\right)$, the set $\left(S_{k}{ }^{\prime}\right)$ forming a non-homogeneous system in $\bar{y}_{1}^{(k)}, \cdots, \bar{y}_{r_{k}}^{(k)}$, if the sets $\left(S_{1}{ }^{\prime}\right), \cdots,\left(S_{k-1}^{\prime}\right)$ have been previously integrated.

\section{A SPECIAL FORM OF INVARIANT RELATIONS}

Suppose equations $(1, \S 1)$ in the canonical form

$$
\begin{array}{ll}
\frac{d x_{i}}{d t}=\frac{\partial F}{\partial y_{i}}, \frac{d y_{i}}{d t}=-\frac{\partial F}{\partial x_{i}} & (i=1, \cdots, m), \\
\frac{d u_{i}}{d t}=\frac{\partial F}{\partial v_{i}}, \frac{d v_{i}}{d t}=-\frac{\partial F}{\partial u_{i}} & (i=1, \cdots, r),
\end{array}
$$

and suppose the equations

$$
u_{1}=u_{2}=\cdot \cdot=u_{r}=v_{1}=v_{2}=\cdot \cdot \cdot=v_{r}=0
$$

a set of invariant relations. Then the functions $\partial F / \partial u_{i}, \partial F / \partial v_{k}$ are of at least the first degree in $u_{i}, v_{i}$, and the equations of variation for $\left(1^{\prime}\right)$ are

$$
\begin{aligned}
\frac{d \xi_{i}}{d t} & =\sum_{k=1}^{m} \frac{\partial^{2} F}{\partial y_{i} \partial x_{k}} \xi_{k}+\sum_{k=1}^{m} \frac{\partial^{2} F}{\partial y_{i} \partial y_{k}} \eta_{k}, \\
\frac{d \eta_{i}}{d t} & =-\sum_{k=1}^{m} \frac{\partial^{2} F}{\partial x_{i} \partial x_{k}} \xi_{k}-\sum_{k=1}^{m} \frac{\partial^{2} F}{\partial x_{i} \partial y_{k}} \eta_{k} \quad(i=1, \ldots,, m) ;
\end{aligned}
$$$$
\frac{d \bar{u}_{i}}{d t}=\sum_{k=1}^{r} \frac{\partial^{2} F}{\partial v_{i} \partial u_{k}} \bar{u}_{k}+\sum_{k=1}^{r} \frac{\partial^{2} F}{\partial v_{i} \partial v_{k}} \bar{v}_{k},
$$$$
\frac{d \bar{v}_{i}}{d t}=-\sum_{k=1}^{r} \frac{\partial^{2} F}{\partial u_{i} \partial u_{k}} \bar{u}_{k}-\sum_{k=1}^{r} \frac{\partial^{2} F}{\partial u_{i} \partial v_{k}} \bar{v}_{k} \quad(i=1, \ldots, r) \text {. }
$$

\section{Periodic ORBITS}

In this paragraph will be outlined a variation of the method of Poincare, for determining the periodic solutions of a differential system, which does not contain a parameter explicitly.

Suppose

$$
\bar{x}_{i}=\varphi_{i}^{0}(t), \varphi_{i}^{0}(0)=x_{i}^{0} \quad(i=1, \cdots, n)
$$

a periodic solution of the system

$$
\frac{d x_{i}}{d t}=X_{i}\left(x_{1}, x_{2}, \cdots, x_{n}\right) \quad(i=1, \cdots, n),
$$


and consider a solution

$$
x_{i}=\varphi_{i}(t), \quad \varphi_{i}(0)=x_{i}^{0}+\beta_{i}
$$$$
(i=1, \cdots \cdot n) \text {; }
$$

the functions $\varphi_{i}$ are analytic in $\beta_{1}, \cdots, \beta_{n}$ for $\left|\beta_{i}\right|<\epsilon$, under the hypotheses of the first paragraph, if $\epsilon$ is sufficiently small, and can be expanded as power series in these variables.*

The expansion of $x_{i}$ is in the form

$$
x_{i}=x_{i}^{0}(t)+\sum_{k=1}^{n} \xi_{i}^{(k)}(t) \beta_{k}+\Phi_{2}^{(i)}\left(t ; \beta_{1}, \cdots, \beta_{n}\right) \quad(i=1, \cdots, n)
$$

where $\Phi_{2}^{(1)}$ is of at least the second degree with respect to $\beta_{1}, \cdots, \beta_{n}$, the coefficients being continuous functions of $t$, and the series converge if $0 \leqq t \leqq \bar{T},\left|\beta_{i}\right|<\epsilon(i=1,2, \cdots, n)$.

The functions $\xi_{i}^{(k)}(t)$ are solutions of the equations of variation for (1),

$$
\frac{d \xi_{i}^{(k)}}{d t}=\sum_{i=1}^{n} \frac{\partial X_{i}}{\partial x_{i}} \xi_{i}^{(k)} \quad(i=1, \cdots, n ; k=1, \cdots, n),
$$

and are such that for $t=0$,

$$
\xi_{i}^{(k)}(0)=\delta_{i}^{(k)}
$$$$
\left(\delta_{i}^{(i)}=1, \quad \delta_{i}^{(k)}=0, \quad k \neq i\right) .
$$

If the generating periodic orbit has the period $T$, the problem of determining periodic orbits of period $T+\tau$ is reduced to that of solving the system of equations

$$
\Delta x_{i}=x_{i}(T+\tau)-x_{i}(0)=0
$$$$
(i=1, \cdots \cdot n) \text {, }
$$

for $\beta_{1}, \cdots, \beta_{n}, \tau$. From (2),

where

$$
\Delta x_{i}=x_{i}^{\prime}(0) \tau+\sum_{k=1}^{n} \Delta \xi_{i}^{(k)} \beta_{k}+\psi_{2}^{(i)}\left(\tau, \beta_{1}, \cdots, \beta_{n}\right),
$$

$$
\Delta \xi_{i}^{(k)}=\xi_{i}^{(k)}(T)-\xi_{i}^{(k)}(0) \quad(i=1, \cdots, n ; k=1, \cdots, n),
$$

and $\psi_{2}^{(1)}$ is of at least the second degree in $\tau, \beta_{1}, \cdots, \beta_{n}$.

Equations (5) can be solved for small values of $\tau, \beta_{1}, \cdots, \beta_{n}$ if not all $n$-rowed determinants of the matrix

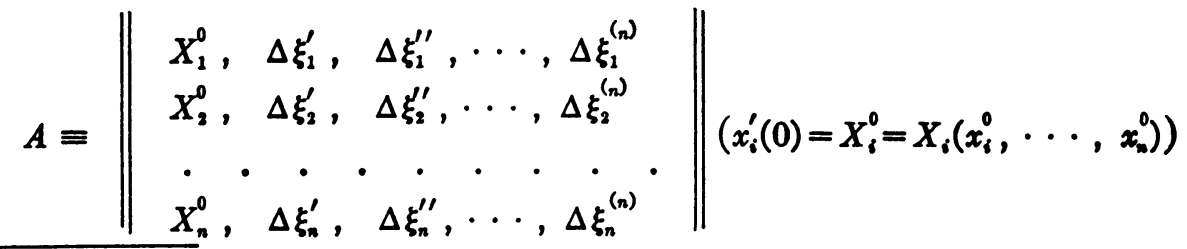

- Les Methodes Nouvelles, vol. 1, Chap. 2. 
are zero. If a certain number of uniform integrals of (1) exist, the rank of $A$ will in general be less than $n$; but in this case* these integrals can be used in place of some of the equations (5).

\section{Certain properties of isosceles triangle solutions With aXis} OF SYMMETRY, IN THE PROBLEM OF THREE RODIES

The equations of motion of the isosceles triangle solutions with axis of symmetry in the problem of three bodies can be given the form

$$
\begin{aligned}
\frac{d r}{d t} & =\frac{\partial F}{\partial R}, \quad \frac{d \zeta}{d t}=\frac{\partial F}{\partial Z}, \\
\frac{d R}{d t} & =-\frac{\partial F}{\partial r}, \quad \frac{d Z}{d t}=-\frac{\partial F}{\partial \zeta}, \\
F & =\frac{1}{2 m^{\prime}}\left(R^{2}+\frac{\theta^{2}}{r^{2}}\right)+\frac{Z^{2}}{2 \bar{M}}-\frac{4 m^{\prime} m_{2}}{r_{0}}-\frac{4 m^{\prime 2}}{r}, \\
\bar{M} & =\frac{4 m^{\prime} m_{2}}{4 m^{\prime}+m_{2}}, \quad r_{0}=\sqrt{\zeta^{2}+(r / 2)^{2}} .
\end{aligned}
$$

In these equations $2 m^{\prime}$ is the common mass of two of the bodies, $m_{2}$ the mass of the third, $r$ the distance between the two equal masses, $r_{0}$ the distance from either to the third, and $\zeta$ is the distance from the third to the center of gravity of the first two.

These equations admit the integral $F=C$, and it was shown by J. Chazy $\dagger$ that if $C<0, \theta \neq 0, r$ must satisfy certain inequalities

$$
0<a \leqq r \leqq \beta .
$$

It can also be shown that positive constants $\bar{A}, \bar{B}$ can be found such that if $\theta \neq 0, C<-\bar{A}$, then $|\zeta|<\bar{B}$ and consequently

$$
0<\gamma<r_{0}<\delta
$$

for certain constants $\gamma, \delta$ and for all values of $t$.

For from the energy integral, if $C=-C^{\prime}$,

$$
\begin{aligned}
& \frac{1}{2 m^{\prime}}\left(R^{2}+\frac{\theta^{2}}{r^{2}}\right)+\frac{Z^{2}}{2 \bar{M}}-\frac{4 m^{\prime} m_{2}}{r_{0}}-\frac{4 m^{\prime 2}}{r}=-C^{\prime}, \\
& \frac{R^{2}}{2 m^{\prime}}+\frac{Z^{2}}{2 \bar{M}}=\frac{4 m^{\prime} m_{2}}{r_{0}}+\frac{4 m^{\prime 2}}{r}-\frac{\theta^{2}}{2 m^{\prime} r^{2}}-C^{\prime} \geqq 0 .
\end{aligned}
$$

- Les Methodes Nouvelles, vol. 1, Chap. 3.

† Bulletin Astronomique, vol. 1, No. 3, 1921. 
Assume $\theta \neq 0$; then

from which

$$
-\frac{1}{r^{2}}-\frac{8 m^{\prime 3}}{\theta^{2} r} \geqq \frac{2 m^{\prime} C^{\prime}}{\theta^{2}}-\frac{8 m^{\prime 2} m_{2}}{\theta^{2} r_{0}},
$$

$$
\begin{aligned}
-\left(\frac{1}{r}-\frac{4 m^{\prime 3}}{\theta^{2}}\right)^{2} & \geqq \frac{2 m^{\prime} C^{\prime}}{\theta^{2}}-\left(\frac{4 m^{\prime 3}}{\theta^{2}}\right)^{2}-\frac{8 m^{\prime 2} m_{2}}{\theta^{2} r_{0}}, \\
\frac{4 m^{\prime 2} m_{2}}{r_{0}} & \geqq \frac{m^{\prime} C^{\prime} \theta^{2}-8 m^{\prime 6}}{\theta^{2}} ;
\end{aligned}
$$

or if $C^{\prime} \theta^{2}-8 m^{\prime}>0$,

$$
|\zeta| \leqq r_{0} \leqq \frac{4 m^{\prime} m_{2} \theta^{2}}{C^{\prime} \theta^{2}-8 m^{\prime 5}} .
$$

If the variable $t$ is replaced by $\theta$, from the relation

equations (1) become

$$
m^{\prime} r^{2} \frac{d \theta}{d t}=\theta, \quad d \theta=\frac{\theta}{m^{\prime} r^{2}} d t,
$$

$$
\begin{array}{ll}
\frac{d r}{d \theta}=\frac{m^{\prime} r^{2}}{\theta} \frac{\partial F}{\partial R}, & \frac{d \zeta}{d \theta}=\frac{m^{\prime} r^{2}}{\theta} \frac{\partial F}{\partial Z}, \\
\frac{d R}{d \theta}=-\frac{m^{\prime} r^{2}}{\theta} \frac{\partial F}{\partial r}, & \frac{d Z}{d \theta}=-\frac{m^{\prime} r^{2}}{\theta} \frac{\partial F}{\partial \zeta},
\end{array}
$$

and these equations admit the multiplier $M=\theta / m^{\prime} r^{2}$, where $M$ is positive and finite so long as the initial conditions are such that

$$
C^{\prime} \theta^{2}-8 m^{\prime 5}>C_{1}^{2}>0 \text {; }
$$

while from the assumption $C^{\prime}<0$ it follows that

$$
r \geqq \frac{\theta^{2}}{2 m^{\prime}\left(8 m^{\prime} m_{2}+4 m^{\prime 2}\right)},
$$

an inequality easily obtained from the energy integral and the inequality $1 / r \geqq 1 / 2 r_{0}$.

It follows that for some region

(D)

$$
0<a_{1}<|r|<b_{1}, \quad|R|<B_{1},
$$

$$
|\zeta|<\bar{B}, \quad|Z|<\bar{C}
$$

for which

$$
C_{2}^{2}>C^{\prime} \theta^{2}-8 m^{\prime 8}>C_{1}^{2}>0,
$$


the variables $r, R, \zeta, Z$ lie in $(D)$ for all values of $t$, and the equations $\left(1^{\prime}\right)$ remain valid; since these equations admit a positive finite multiplier the solutions possess compiete stability.*

From (1) we obtain

$$
\frac{d \zeta}{d t}=\frac{Z}{\bar{M}}, \quad \frac{d Z}{d t}=-\frac{4 m^{\prime} m_{2}}{r^{2}} \zeta,
$$

or if $a=\sqrt{4 m^{\prime} m_{2} / \bar{M}}$,

From (3),

$$
\frac{d^{2} \zeta}{d t^{2}}+\frac{a^{2}}{r_{0}^{3}} \zeta=0
$$

Consider an equation

$$
\frac{a^{2}}{\delta^{3}}<\frac{a^{2}}{r_{0}^{3}}<\frac{a^{2}}{\gamma^{r}} .
$$

$$
\frac{d^{2} \zeta}{d t^{2}}+\varphi(t, \zeta) \zeta=0,
$$

in which

$$
A^{2}<\varphi(t, \zeta)<B^{2}, \quad|\zeta|<\epsilon,
$$

for all values of $t, A$ and $B$ being certain positive constants, and $\varphi$ continuous in its arguments. Let $z_{1}, z_{2}$ be solutions of the equations

Then

$$
\begin{aligned}
& \frac{d^{2} z_{1}}{d t^{2}}+B^{2} z_{1}=0, \\
& \frac{d^{2} z_{2}}{d t_{2}}+A^{2} z_{2}=0 .
\end{aligned}
$$

$$
\begin{aligned}
& z_{1} \zeta^{\prime \prime}-\zeta z_{1}^{\prime \prime}=\zeta z_{1}\left[B^{2}-\varphi(t, \zeta)\right], \\
& \zeta z_{2}^{\prime \prime}-z_{2} \zeta^{\prime \prime}=\zeta z_{2}\left[\varphi(t, \zeta)-A^{2}\right] .
\end{aligned}
$$

If $z_{1}, z_{2}, \zeta$ satisfy the initial conditions

then

$$
\begin{gathered}
z_{1}(0)=z_{2}(0)=\zeta(0)=0, \\
z_{1}{ }^{\prime}(0)>0, \quad z_{2}{ }^{\prime}(0)>0, \quad \zeta^{\prime}(0)>0,
\end{gathered}
$$

$$
\left[z_{1} \zeta^{\prime}-\zeta z_{1}^{\prime}\right]_{0}^{t_{1}}=\int_{0}^{t_{1}}\left[B^{2}-\varphi(t, \zeta)\right] z_{1} \zeta d t,
$$

- Poincare, Les Methodes Nouvelles, vol. 3, p. 141. For the method of Poincare can be applied to prove that if $P\left\{r_{0}, \zeta_{0}, R_{0}, Z_{0}\right\}$ is any point of $(D), \delta$ any region surrounding $P, Q$ an arbitrary point of $\delta, \theta_{0}$ a given value of $\theta$, the probability that $(r, \zeta, R, Z)$ will lie in $\delta$ only a finite number of times with $\theta=\theta_{0}+2 k \pi$ is arbitrarily small ( $k$ an integer) if $(r, \zeta, R, Z)=Q, \theta=\theta_{0}$. 
or if $z_{1}\left(t_{1}\right)=0, t_{1}$ being the first zero of $z_{1}(t)$ after $t=0$,

$$
-\zeta\left(t_{1}\right) z_{1}^{\prime}\left(t_{1}\right)>0
$$

and since $z_{1}^{\prime}\left(t_{1}\right)<0, \zeta\left(t_{1}\right)>0$. In the same manner we obtain

$$
\zeta(\bar{t}) z_{2}^{\prime}(\bar{t})-z_{2}(\bar{t}) \zeta^{\prime}(\bar{t})=\int_{0}^{\bar{t}}\left[\varphi(t, \zeta)-A^{2}\right] z_{2} \zeta d t,
$$

and if $\zeta(\bar{t})=0, \bar{t}$ being the first zero of $\zeta(t)$ greater than $t=0$,

$$
-\zeta^{\prime}(\bar{t}) z_{2}(\bar{t})>0 \text {, }
$$

from which $z_{2}(\bar{t})>0$. Consequently if $\zeta(0)=0$, the first following zero of $\zeta(t)$ must lie between the first zero of $z_{1}(t)$ and that of $z_{2}(t)$. If this zero is $\bar{t}$,

$$
\frac{\pi}{B}<\bar{t}<\frac{\pi}{A}
$$

applying this result to equation (4), with conditions (5),

$$
\frac{\pi \gamma^{3 / 2}}{a}<\bar{t}<\frac{\pi \delta^{3 / 2}}{a}
$$

From (6) it follows also that if $0<t<\pi / B$,

consequently if $0<t_{1}<t_{2}<\pi / B$,

$$
\frac{z_{1} \zeta^{\prime}-\zeta z_{1}^{\prime}}{z_{1}^{2}}>0
$$

$$
\left[\frac{\zeta}{z_{1}}\right]_{t_{1}}^{t_{2}}=\int_{t_{1}}^{t_{2}} \frac{z_{1} \zeta^{\prime}-\zeta z_{1}^{\prime}}{z_{1}^{2}} d t>0 .
$$

Now suppose $z_{1}^{\prime}(0)=\zeta^{\prime}(0)$; for small values of $t$,

$$
\begin{aligned}
& \zeta=\zeta_{0}^{\prime} t+t^{2} \psi(t), \\
& z_{1}=\frac{\zeta_{0}^{\prime}}{B} \sin B t .
\end{aligned}
$$

From (9),

$$
\frac{\zeta\left(t_{2}\right)}{z_{1}\left(t_{2}\right)}>\frac{\zeta_{0}^{\prime} t_{1}+t_{1}^{2} \psi\left(t_{1}\right)}{\frac{\zeta_{0}^{\prime}}{B} \sin B t_{1}} .
$$


Taking the limit of the expression on the right of (10) as $t_{1}$ approaches zero,

$$
\frac{\zeta\left(t_{2}\right)}{z_{1}\left(t_{2}\right)}>1
$$

since the right member of (10) decreases as $t_{1}$ approaches zero.

An exactly analogous argument shows that if $\zeta(t)>0,0<t<\bar{i}$, and $z_{2}^{\prime}(0)=\zeta^{\prime}(0)$,

or

$$
\frac{z_{2}(t)}{\zeta(t)}>1
$$

$$
\begin{array}{ll}
z_{1}\left(t_{2}\right)<\zeta\left(t_{2}\right), & 0<t_{2}<\frac{\pi}{B}, \\
\zeta(t)<z_{2}(t), & 0<t<\bar{t} .
\end{array}
$$

Consequently* if $\bar{\zeta}$ is the maximum value of $\zeta$ on the interval $0<t<\bar{t}$,

$$
\frac{\zeta_{0}^{\prime}}{B} \leqq \bar{\zeta} \leqq \frac{\zeta_{0}^{\prime}}{A}
$$

or

$$
\frac{1}{B} \leqq \frac{\bar{\zeta}}{\zeta_{0}^{\prime}} \leqq \frac{1}{A} .
$$

5. Periodic isosceles triangle solutions

Suppose the function $F$ in equations $(1, \S 4)$ expanded in powers of $\zeta$; since

$$
\begin{aligned}
\frac{1}{r_{0}} & =\frac{2}{r}\left[1+\left(\frac{2 \zeta}{r}\right)^{2}\right]^{-1 / 2} \\
& =\frac{2}{r}\left[1-\frac{1}{2}\left(\frac{2 \zeta}{r}\right)^{2}+\frac{1 \cdot 3}{2 \cdot 4}\left(\frac{2 \zeta}{r}\right)^{4}-\frac{1 \cdot 3 \cdot 5}{2 \cdot 4 \cdot 6}\left(\frac{2 \zeta}{r}\right)^{r}+\cdots\right]
\end{aligned}
$$

we obtain

$$
\begin{aligned}
F= & \frac{1}{2 m^{\prime}}\left(R^{2}+\frac{\theta^{2}}{r^{2}}\right)-\frac{4 m^{\prime 2}+8 m^{\prime} m_{2}}{r} \\
& +\frac{Z^{2}}{2 \bar{M}}+\frac{16 m^{\prime} m_{2}}{r^{3}} \zeta^{2} \\
& \quad-\frac{8 m^{\prime} m_{2}}{r}\left[\frac{1 \cdot 3}{2 \cdot 4}\left(\frac{2 \zeta}{r}\right)^{4}-\frac{1 \cdot 3 \cdot 5}{2 \cdot 4 \cdot 6}\left(\frac{2 \zeta}{r}\right)^{0}+\cdots\right] \\
= & F_{0}+F_{2}+F_{1},
\end{aligned}
$$

- That $\zeta(t)$ must have at least one zero can be shown easily from the equation

$$
\left[z_{2}^{\prime}-z_{2} 5^{\prime}\right]_{i_{1}}^{t_{2}}=\int_{t_{1}}^{t_{2}}\left[\varphi-A^{2}\right] \zeta z_{2} d t
$$

by the method above, if $z_{2}\left(t_{1}\right)=\zeta\left(t_{1}\right)>0, z_{2}^{\prime}\left(t_{1}\right)=\zeta^{\prime}\left(t_{1}\right)$. 
where $F_{0}$ is independent of $\zeta, Z, F_{2}$ is of the second degree, and $F_{1}$ of at least the fourth degree with respect to these variables. The equations $\zeta=Z=0$ form evidently a set of invariant relations for equations $(1, \S 4)$. If $\mu=8 m_{2}+4 m^{\prime}$,

$$
F_{0}=\frac{1}{2 m^{\prime}}\left(R^{2}+\frac{\theta^{2}}{r^{2}}\right)-\frac{m^{\prime} \mu}{r},
$$

and equations $(1, \S 4)$, reduced by the relations $\zeta=Z=0$, can be integrated by the method of Jacobi. A complete integral of the partial differential equation

$$
\frac{1}{2 m^{\prime}}\left[\left(\frac{\partial S}{\partial r}\right)^{2}+\frac{\theta^{2}}{r^{2}}\right]-\frac{m^{\prime} \mu}{r}=-\frac{m^{\prime 3} \mu^{2}}{2 L^{2}}
$$

is the function

$$
S=\int \sqrt{-\frac{\theta^{2}}{r^{2}}+\frac{2 m^{\prime 2} \mu}{r}-\frac{m^{\prime 4} \mu^{2}}{L^{2}}} d r,
$$

where $\theta, L$ are constants. If

equations $(1, \S 4)$ become

$$
R=\frac{\partial S}{\partial r}, \quad l=\frac{\partial S}{\partial L},
$$

$$
\begin{aligned}
\frac{d l}{d t} & =\frac{\partial F}{\partial L}, \quad \frac{d \zeta}{d t}=\frac{\partial F}{\partial Z}, \\
\frac{d L}{d t} & =-\frac{\partial F}{\partial l}, \quad \frac{d Z}{d t}=-\frac{\partial F}{\partial \zeta}, \\
F & =-\frac{m^{\prime 3} \mu^{2}}{2 L^{2}}+F_{2}+F_{1} .
\end{aligned}
$$

One might be tempted to employ the method of $\$ 3$ to determine periodic solutions of (4). Suppose

$$
\begin{aligned}
\Delta \zeta & =\zeta(T+\tau)-\zeta(0), \\
\Delta Z & =Z(T+\tau)-Z(0), \\
\psi & =l(T+\tau)-n_{0} T, \quad n_{0}=\frac{m^{\prime 3} \mu^{2}}{L^{3}}, \\
\Delta L & =L(T+\tau)-L(0),
\end{aligned}
$$

if the reduced system has a periodic solution of period $T=2 \pi / n_{0}$. Since $F=C$ is an integral for (4), the equation $\Delta L=0$ can be omitted, and equations

$$
\Delta \zeta=\Delta Z=\psi=0
$$


can be solved for $\delta \zeta^{\circ}, \delta Z^{0}, \delta L^{0}$ in terms of $\tau$ if the determinant

$$
J=\left|\begin{array}{ccc}
T \frac{\partial n}{\partial L} & 0 & 0 \\
0 & \Delta \bar{\zeta}_{1} & \Delta \bar{\zeta}_{2} \\
0 & \Delta \bar{Z}_{1} & \Delta \bar{Z}_{2}
\end{array}\right|
$$

is different from zero; $\bar{\zeta}_{i}, \bar{Z}_{i}$ are solutions of the equations of variation for the system (4),

$$
\frac{d \bar{\zeta}}{d t}=\frac{\bar{Z}}{\bar{M}}, \quad \frac{d \bar{Z}}{d t}=-\frac{32 m^{\prime} m_{2}}{r^{3}} \bar{\zeta},
$$

from which

$$
\frac{d^{2} \bar{\zeta}}{d t^{2}}+\frac{32 m^{\prime} m_{2}}{\bar{M} r^{3}} \bar{\zeta}=0 .
$$

The determinant $J$ can vanish only if at least one of the characteristic exponents of (5) is of the form*

$$
a=\frac{2 \kappa \pi i}{T}=\kappa n i \text {, }
$$

where $\kappa$ is an integer. A simple calculation shows that

$$
\frac{a}{n}= \pm i \sqrt{\frac{2\left(m_{2}+4 m^{\prime}\right)}{2 m_{2}+m^{\prime}}}
$$

if the eccentricity is zero for the reduced orbit. If $a / n=k i$,

$$
\frac{m^{\prime}}{m_{2}}=\frac{2\left(\kappa^{2}-1\right)}{8-\kappa^{2}} \text {. }
$$

Now since $m^{\prime}, m_{2}$ are positive, the only possible value for $\kappa$ is 2 and

$$
\frac{m^{\prime}}{m_{2}}=\frac{3}{2} \text {. }
$$

The characteristic exponents for the system (5) are continuous functions of the eccentricity $e$ in a region $|e|<h$, for some $h>0$, hence if $J \neq 0$ for $e=0, J \neq 0$ for an interval $|e|<h_{1} \leqq h$. Consequently if the equation (7) is not satisfied, periodic solutions of period $T^{\prime}=T+\tau$ must exist for a certain region $|\tau|<\epsilon ;|e|<h_{1}$.

* Les Methodes Nouvelles, vol. 1, p. 181. 
But these are necessarily isosceles triangle solutions, for which $\zeta=Z=0$. In fact periodic isosceles triangle solutions in the plane exist, with $\zeta=Z=0$, and for these solutions the eccentricity of the relative elliptic movement can have any value between zero and one. From the equations satisfied by a periodic solution it is seen that there can be only one family of periodic isosceles triangle solutions, hence no periodic solutions except the plane isosceles triangle solutions, in general. For any other family of periodic solutions of the type considered, the ratio $a /$ in is necessarily commensurable, where $\pm a$ are the characteristic exponents, $2 \pi / n$ the period of the generating plane isosceles triangle solution.

The plane isosceles triangle solutions (for which $\zeta=Z=0$ ) can be still further particularized by substituting $\theta=m^{\prime} \sqrt{\mu r_{1}}$, for which value the equations $r=r_{1}, R=0$ form a set of invariant relations. If we make the change of variables $r=r_{1}+\eta$, the equations of motion, for small values of $\eta$ and $\zeta$, can be written

$$
\frac{d}{d t}\left(\frac{\partial T}{\partial \eta^{\prime}}\right)=\frac{\partial U}{\partial \eta}, \frac{d}{d t}\left(\frac{\partial T}{\partial \zeta^{\prime}}\right)=\frac{\partial U}{\partial \zeta},
$$

if

$$
\begin{aligned}
& T=\frac{1}{2}\left[m^{\prime} \eta^{\prime 2}+\bar{m} \zeta^{\prime 2}\right], \\
& U=-\frac{m^{\prime} \mu}{2 r_{1}^{3}} \eta^{2}-\frac{16 m^{\prime} m_{2}}{r_{1}^{3}} \zeta^{2}+\phi_{3}(\eta, \zeta) .
\end{aligned}
$$

It follows immediately from the classical theorem of Lagrange that the generating periodic solution is stable (within the family of isosceles triangle solurtions).

\section{Certain PROPERTIES OF THE CHARACTERISTIC EXPONENTS}

Suppose the movement of a material system defined by the equations

$$
\begin{aligned}
& \frac{d x_{i}}{d t}=\frac{\partial F}{\partial y_{i}}, \quad \frac{d y_{i}}{d t}=-\frac{\partial F}{\partial x_{i}} \quad(i=1,2, \cdots, m), \\
& \frac{d u_{k}}{d t}=\frac{\partial F}{\partial v_{k}}, \quad \frac{d v_{k}}{d t}=-\frac{\partial F}{\partial u_{k}} \quad(k=1,2, \cdots, r),
\end{aligned}
$$

where $F$ is periodic, of period $T$ in $t$, and analytic in its remaining $(2 m+2 r)$ variables within a certain domain $D$. If a periodic solution of period $T$, lying within $D$, is defined by the equations

$$
\begin{array}{lll}
x_{i}=x_{i}^{0}(t), & y_{i}=y_{i}^{0}(t) & (i=1, \cdots, m), \\
u_{k}=u_{k}^{0}(t), & v_{k}=v_{k}^{0}(t) & (k=1, \cdots, r),
\end{array}
$$


many of the properties of orbits within a certain neighborhood of $(c)$ can be obtained from a study of the equations of variation corresponding to this orbit.

Suppose $u_{k}^{0}(t) \equiv v_{k}^{0}(t) \equiv 0, k=1, \cdots, r, \partial F / \partial t=0$, for $(c)$, and in addition suppose $u_{k}=v_{k}=0$ a set of invariant relations. It has already been seen that under these conditions the equations of variation break up into two or more distinct systems $(\$ \$ 1,2)$. The characteristic exponents of each system are also characteristic exponents of $(c)$.

If the variables $u_{i}$ are integrals of equations (1), then on account of the relations $\partial F / \partial v_{k} \equiv 0, k=1, \cdots, r$, the equations of variation have the form

(2)

(a)

$$
\frac{d \xi_{i}}{d t}=\sum_{k=1}^{m} \frac{\partial^{2} F}{\partial y_{j} \partial x_{k}} \xi_{k}+\sum_{k=1}^{m} \frac{\partial^{2} F}{\partial y_{i} \partial y_{k}} \eta_{k}+\sum_{k=1}^{r} \frac{\partial^{2} F}{\partial y_{i} \partial u_{k}} a_{k},
$$

(B) $\frac{d a_{i}}{d t}=0$,

$$
\frac{d \eta_{i}}{d t}=-\sum_{k=1}^{m} \frac{\partial^{2} F}{\partial x_{i} \partial x_{k}} \xi_{k}-\sum_{k=1}^{m} \frac{\partial^{2} F}{\partial x_{i} \partial y_{k}} \eta_{k}-\sum_{k=1}^{\infty} \frac{\partial^{2} F}{\partial x_{i} \partial u_{k}}\left(\begin{array}{l}
(i=1, \ldots, m) ; \\
d u_{i}
\end{array}\right.
$$

(r) $\frac{d \bar{v}_{i}}{d t}=-\sum_{k=1}^{m} \frac{\partial^{2} F}{\partial u_{i} \partial x_{k}} \xi_{k}-\sum_{k=1}^{m} \frac{\partial^{2} F}{\partial u_{i} \partial y_{k}} \eta_{k}-\sum_{k=1}^{\infty} \frac{\partial^{2} F}{\partial u_{i} \partial u_{k}} a_{k}$

$$
(i=1, \cdots, r) \text {. }
$$

These equations can be integrated in the order $(\beta),(a),(\gamma)$, the systems $(a),(\gamma)$ being considered as non-homogeneous systems.

Suppose given a non-homogeneous linear system with periodic coefficients, which are also assumed analytic:

for which

$$
\frac{d x_{i}}{d t}=\sum_{k=1}^{n} a_{i k} x_{k}+f_{i} \quad(i=1, \cdots, n),
$$

$$
\sum_{i=1}^{n} a_{i i}=0 \text {. }
$$

Suppose an independent set of solutions of the homogeneous system corresponding to (3) represented by the set of columns of the matri*

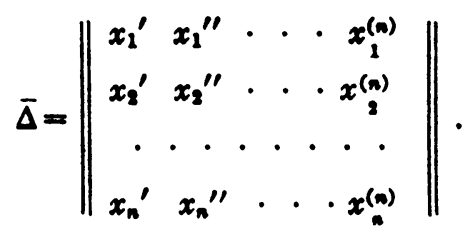


Then $\Delta=\left|x_{i}^{(2)}\right|$ is constant and different from zero, as a consequence of the classical theorems on linear differential systems. If $\Delta_{i}^{(k)}$ is the cofactor of $x_{i}^{(\mathbf{k})}$ in $\Delta$, an arbitrary solution of equations (3) can be represented in the form

(4) $x_{i}=w^{\prime} x_{i}{ }^{\prime}+w^{\prime \prime} x_{i}^{\prime \prime}+\cdots+w^{(n)} x_{i}^{(n)}+\sum_{k=1}^{n} c_{k} x_{i}^{(k)} \quad(i=1, \cdots, n)$ in which

$$
w^{(k)}=\frac{1}{\Delta} \int_{0}^{t}\left[f_{1}(z) \Delta_{1}^{(k)}(z)+f_{2}(z) \Delta_{2}^{(k)}(z)+\cdots+\begin{array}{r}
\left.+f_{n}(z) \Delta_{n}^{(k)}(z)\right] d z \\
(k=1,2, \cdots, n) .
\end{array}\right.
$$

Now the functions $x_{i}^{(k)}(t)$ are of the form

$$
x_{i}^{(k)}(t)=e^{a k t} \varphi_{i}^{(k)}(t) \quad(i, k=1, \cdots, n),
$$

where $\varphi_{i}^{(k)}$ is a polynomial in $t$, of degree at most $n$, whose coefficients are periodic, of period $T$ in $t$. It follows that $\Delta_{i}^{(k)}(t)$ has a similar form. For instance,

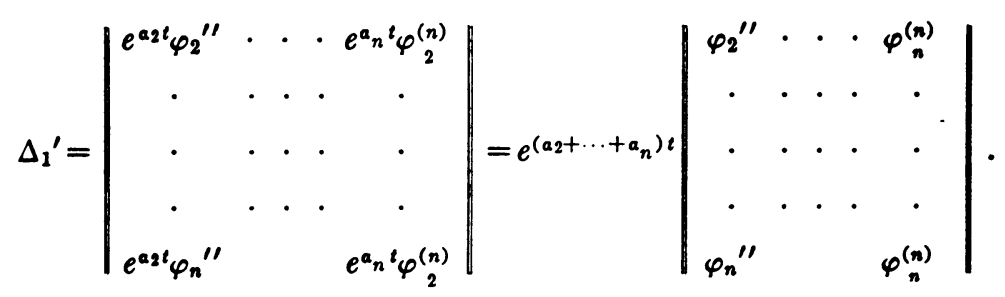

If equations (3) are those of a dynamical system,

whence

$$
a_{1}+a_{2}+\cdots+a_{n}=0 \text {, }
$$

$$
\Delta_{1}{ }^{\prime}=e^{-a_{1}{ }^{t} \psi_{1}{ }^{\prime}(t),}
$$

$\psi_{1}{ }^{\prime}(t)$ being a polynominal in $t$ with periodic coefficients, of degree at most $(n-1) .^{*}$

In general,

$$
\Delta_{i}^{(k)}=e^{-a k t} \psi_{i}^{(k)}(t) \quad(i, k=1, \cdots, n) .
$$

Equations (2), (a) are of the type of (3), with $n=2 m$, and

$$
f_{1}=\sum_{k=1}^{r} \frac{\partial^{2} F}{\partial y_{i} \partial u_{k}} a_{k} \quad(i=1, \cdots, m),
$$

- This degree is less than $n$, since each set of functions $\Delta_{i}^{k}(t)(i=1, \cdots, n)$ is a solution of the differential system adjoint to the homogeneous system of (3). 


$$
f_{1}=-\sum_{k=1}^{r} \frac{\partial^{2} F}{\partial x_{i} \partial u_{k}} \tilde{u}_{k}
$$

$$
(i=m+1, \cdot \cdot, n) .
$$

If these functions are substituted in (5), we obtain

$$
\begin{aligned}
w^{(k)}(t) & =\frac{1}{\Delta} \int_{0}^{t} e^{-a_{k z}}\left[f_{1}(z) \psi_{1}^{(k)}(z)+\cdots+f_{n}(z) \psi_{n}^{(k)}(z)\right] d z & \\
& =\frac{1}{\Delta} \int_{0}^{t} e^{-a_{k z} \phi^{(k)}}(z) d z & (k=1, \cdots, n) .
\end{aligned}
$$

The function $\phi^{(k)}(z)$ is a polynominal in $z$ with periodic coefficients, of degree at most $(n-1)$, consequently*

$$
w^{(k)}(t)=e^{-a_{k} t} \psi^{(k)}(t),
$$

$\psi^{(k)}(t)$ being a polynomial with periodic coefficients, of degree at most $n$.

Substituting these functions in (4), we obtain

$$
x_{i}=\varphi_{i}^{\prime} \psi^{\prime}+\varphi_{i}^{\prime \prime} \psi^{\prime \prime}+\cdots \cdot+\varphi_{i}^{(n)} \psi^{(n)}+\sum_{k=1}^{n} c_{i}^{k} x_{i}^{(k)}(i=1, \cdots, n)
$$

and all the exponents different from zero, in the expression for $x_{i}$, occur in the sum $\sum_{k=1}^{n} C_{k} x_{i}^{(\mathbf{k})}$ and are, therefore, characteristic exponents of the homogeneous system of $(a)$.

The integration of equations $(\gamma)$ gives

$$
\bar{v}_{i}=-\sum_{k=1}^{m} \int_{0}^{t} \frac{\partial^{2} F}{\partial u_{i} \partial x_{k}} \xi_{k} d t-\sum_{k=1}^{m} \int_{0}^{t} \frac{\partial^{2} F}{\partial u_{i} \partial y_{k}} \eta_{k} d t-\sum_{k=1}^{r} \int_{0}^{t} \frac{\partial^{2} F}{\partial u_{i} \partial u_{k}} a_{k} d t,
$$

and from a discussion analogous to that already given it follows that the only exponents in this expression not of the form $a=2 \pi m i / T$ ( $m$ an integer) are exponents of the functions $\xi_{i}, \eta_{i}$.

* For each coefficient of the polynomial $\phi^{k}(z)$ is analytic, hence can be expanded in a Fourier series which converges absolutely and uniformly for all real values of $z$; and the integral can be evaluated by the term-by-term integration of these series:

$$
\begin{aligned}
\int_{0}^{t} e^{-a_{k z} \phi(k)}(z) d z & =\sum_{s=1}^{p} \int_{0}^{t}\left\{z^{s} e^{-a_{k} z} \sum_{r=0}^{\infty}\left[A_{r} \cos \frac{2 r \pi z}{T}+B_{r} \sin \frac{2 r \pi z}{T}\right]\right\} d z \\
& =\sum_{s=1}^{p}\left\{\sum_{r=0}^{\infty}\left[A_{r} \int_{0}^{t} e^{-a_{k z} z^{s}} \cos \frac{2 r \pi z}{T} d z+B_{r} \int_{0}^{t} e^{-a_{k} z z^{s}} \sin \frac{2 r \pi z}{T} d z\right]\right\} .
\end{aligned}
$$

Each integral can be expressed as a sum of terms of the same form as the integrand, of degree at most one greater in $t$; the numerical coefficients introduced are bounded, for all values of $r$, hence the series of integrated terms converges absolutely and uniformly and when expressed as a poylnomial in $t$, multiplied by $e^{-a t}$, is of the form (7). 
It is an immediate consequence that the characteristic exponents different from zero can be calculated either from the original system $(2 ; a, \beta, \gamma)$ or from the reduced system $(2, a)$, which are the equations of variation for the system obtained from (1) by substituting $u_{i}=c_{i}, \bar{u}_{i}=0$, and ignoring the coördinates $v_{1}, \cdots, v_{r}$.

Another property, of importance in the calculation of the characteristic exponents, is their invariance under an analytic change of variables which does not involve the time.*

\section{REDUCTION OF THE EQUATIONS OF MOTION IN THE NEIGHBORHOOD OF THE STRAIGHT LINE SOLUTIONS}

In the following paragraphs we shall apply some of the preceding results to the study of orbits in the neighborhood of the straight line solutions, and the equilateral triangle solutions, respectively, in the problem of three bodies. The notion of invariant relation appears especially convenient.

If three masses $m_{0}, m_{1}, m_{2}$ have the coördinates and components of momentum

$$
\begin{gathered}
m_{0}:\left(x_{1}, x_{2}, x_{3}, y_{1}, y_{2}, y_{3}\right), \\
m_{1}:\left(x_{1}, x_{5}, x_{6}, y_{1}, y_{6}, y_{6}\right), \\
m_{2}:\left(x_{7}, x_{8}, x_{9}, y_{7}, y_{8}, y_{9}\right), \\
y_{i}=m_{0} \frac{d x_{i}}{d t} \\
y_{i}=m_{1} \frac{d x_{i}}{d t} \\
y_{i}=m_{2} \frac{d x_{i}}{d t}
\end{gathered}
$$$$
(i=4,5,6)
$$$$
(i=7,8,9) \text {, }
$$

and if these masses move subject to their mutual attraction according to Newton's law, the equations of motion are

$$
\begin{aligned}
\frac{d x_{i}}{d t} & =\frac{\partial F}{\partial y_{i}}, \frac{d y_{i}}{d t}=-\frac{\partial F}{\partial x_{i}} \\
F & =\frac{1}{2 m_{0}} \sum_{i=1}^{3} y_{i}^{2}+\frac{1}{2 m_{1}} \sum_{i=1}^{0} y_{i}^{2}+\frac{1}{2 m_{2}} \sum_{i=7}^{0} y_{i}^{2}-U, \\
U & =\frac{m_{1} m_{2}}{r_{0}}+\frac{m_{2} m_{0}}{r_{1}}+\frac{m_{1} m_{2}}{r_{2}} .
\end{aligned}
$$

- For transformations involving the time, see Poincare, Les Mathodes Nouvelles, vol. 1, p. 199. 
Here $r_{0}, r_{1}, r_{2}$ are the distances separating the masses $\left(m_{1}, m_{2}\right),\left(m_{2}, m_{0}\right)$, $\left(m_{0}, m_{1}\right)$, respectively. If the center of gravity is taken as the origin, introducing the notation

$$
\begin{aligned}
& \lambda=\frac{m_{1}}{m_{0}+m_{1}}, \quad \mu=\frac{m_{0}}{m_{0}+m_{1}}, M=m_{0}+m_{1}+m_{2}, \\
& g=\frac{M}{m_{2}\left(m_{0}+m_{1}\right)}, \quad h=\frac{m_{0}+m_{1}}{m_{0} m_{1}}, \\
& \bar{x}_{1}=x_{4}-x_{1}, \quad \bar{x}_{2}=x_{6}-x_{2}, \quad \bar{x}_{3}=x_{6}-x_{3}, \\
& \bar{x}_{4}=g m_{2} x_{7}, \quad \bar{x}_{6}=g m_{2} x_{8}, \quad \bar{x}_{8}=g m_{2} x_{0}, \\
& \bar{y}_{i}=\frac{1}{h} \frac{d \bar{x}_{i}}{d t} \quad(i=1,2,3), \\
& \bar{y}_{i}=\frac{1}{g} \frac{d \bar{x}_{i}}{d t} \quad(i=4,5,6),
\end{aligned}
$$

we obtain the equations of motion

(5)

$$
\begin{aligned}
& \quad \frac{d \bar{x}_{i}}{d t}=\frac{\partial F}{\partial \bar{y}_{i}}, \quad \frac{d \bar{y}_{i}}{d t}=-\frac{\partial F}{\partial \bar{x}_{i}} \quad(i=1,2, \ldots, 6) ; \\
& F=\frac{h}{2}\left(\bar{y}_{1}^{2}+\bar{y}_{2}^{2}+\bar{y}_{3}^{2}\right)+\frac{g}{2}\left(\bar{y}_{1}^{2}+\bar{y}_{5}^{2}+\bar{y}_{6}^{2}-U,\right. \\
& r_{6}^{2}=\left(\bar{x}_{4}-\mu \bar{x}_{1}\right)^{2}+\left(\bar{x}_{5}-\mu \bar{x}_{2}\right)^{2}+\left(\bar{x}_{6}-\mu \bar{x}_{3}\right)^{2}, \\
& r_{1}^{2}=\left(\bar{x}_{4}+\lambda \bar{x}_{1}\right)^{2}+\left(\bar{x}_{6}+\lambda \bar{x}_{2}\right)^{2}+\left(\bar{x}_{6}+\lambda \bar{x}_{3}\right)^{2}, \\
& r_{2}^{2}=\bar{x}_{1}^{2}+\bar{x}_{2}^{2}+\bar{x}_{3}^{2} .
\end{aligned}
$$

An examination of equations (1) shows that the relations

$$
x_{8}=x_{6}=x_{9}=y_{3}=y_{6}=y_{9}=0
$$

imply the relations

$$
\frac{d x_{0}}{d t}=\frac{d x_{6}}{d t}=\frac{d x_{0}}{d t}=\frac{d y_{3}}{d t}=\frac{d y_{0}}{d t}=\frac{d y_{0}}{d t}=0,
$$

and consequently, from the principle stated by Poincare ( $\$ 1$ ), equations (6) form a set of invariant relations. Similarly, the equations

$$
x_{2}=x_{6}=x_{8}=y_{2}=y_{6}=y_{8}=0
$$


are a set of invariant relations; the relations $(6)$ and $\left(6^{\prime}\right)$ together form a set which characterizes a movement on a fixed straight line.

Suppose $p$ a mobile straight line through the center of gravity, taken as the origin, and let $x_{0}{ }^{\prime}, x_{1}{ }^{\prime}, x_{2}{ }^{\prime}$ be the coördinates of $m_{0}, m_{1}, m_{2}$ respectively on $p, m_{1}$ lying between $m_{0}$ and $m_{2}$. Suppose

$$
x_{1}{ }^{\prime}=-a x_{0}{ }^{\prime}, \quad x_{2}{ }^{\prime}=-\beta x_{0}{ }^{\prime} .
$$

Then if

it follows that

$$
\rho_{0}^{\prime}=\left|x_{0}^{\prime}\right|, \quad \rho_{1}^{\prime}=\left|x_{1}^{\prime}\right|, \quad \rho_{2}^{\prime}=\left|x_{2}^{\prime}\right|,
$$

$$
r_{0}=(\beta-a) \rho_{0}^{\prime}, \quad r_{1}=(1+\beta) \rho_{0}^{\prime}, \quad r_{2}=(1+a) \rho_{0}^{\prime} .
$$

If equations (6) are satisfied, and the masses $m_{i}$ lie on the line $p$ for all values of the time, while $a, \beta$ are certain constants, then it can be shown* that

Or if

$$
\begin{gathered}
a(1+\beta)^{-2}-\beta(1+a)^{-2}+(\beta-a)^{-2}=0, \\
m_{0}-m_{1} a-m_{2} \beta=0 .
\end{gathered}
$$

$$
\begin{gathered}
\beta-a=(1+a) \nu, \\
m_{1}(1+\nu)^{2}\left(1-\nu^{3}\right)-m_{0} \nu^{2}\left[(1+\nu)^{3}-1\right]+m_{2}\left[(1-\nu)^{3}-\nu^{3}\right]=0,
\end{gathered}
$$

which is the equation of Laplace.

If the polar coördinates are employed

and

$$
\begin{array}{lll}
x_{1}=\rho_{0}{ }^{\prime} \cos \theta_{0}{ }^{\prime}, & x_{4}=\rho_{1}{ }^{\prime} \cos \theta_{1}{ }^{\prime}, & x_{7}=\rho_{2}{ }^{\prime} \cos \theta_{2}{ }^{\prime}, \\
x_{2}=\rho_{0}{ }^{\prime} \cos \theta_{0}{ }^{\prime}, & x_{5}=\rho_{1}{ }^{\prime} \cos \theta_{1}{ }^{\prime}, & x_{8}=\rho_{2}{ }^{\prime} \sin \theta_{2}{ }^{\prime}
\end{array}
$$

$$
F=\sum_{i=1}^{3} \frac{1}{2 m_{i}}\left(R_{i}^{\prime 2}+\frac{\theta_{i}{ }^{2}}{\rho_{i}^{\prime 2}}\right)-U
$$

equations (1) become

$$
\begin{aligned}
\frac{d \rho_{i}{ }^{\prime}}{d t}=\frac{\partial F}{\partial R_{i}^{\prime}}, & \frac{d \theta_{i}^{\prime}}{d t}=\frac{\partial F}{\partial \theta_{i}^{\prime}}, \\
\frac{d R_{i}^{\prime}}{d t}=-\frac{\partial F}{\partial \rho_{i}^{\prime}}, & \frac{d \theta_{i}^{\prime}}{d t}=-\frac{\partial F}{\partial \theta_{i}^{\prime}} \quad(i=0,1,2) .
\end{aligned}
$$

Consider the relations

$$
\begin{aligned}
& \epsilon \pi+\theta_{0}^{\prime}-\theta_{1}^{\prime}=0, \quad \frac{d \theta_{0}^{\prime}}{d t}-\frac{d \theta_{1}^{\prime}}{d t}=0, \quad \rho_{1}^{\prime}-|a| \rho_{0}^{\prime}=0, \quad \frac{d \rho_{1}^{\prime}}{d t}-|a| \frac{d \rho_{0}^{\prime}}{d t}=0, \\
& \pi+\theta_{0}{ }^{\prime}-\theta_{2}{ }^{\prime}=0, \quad \frac{d \theta_{0}{ }^{\prime}}{d t}-\frac{d \theta_{2}{ }^{\prime}}{d t}=0, \quad \rho_{2}{ }^{\prime}-\beta \rho_{0}{ }^{\prime}=0, \quad \frac{d \rho_{2}^{\prime}}{d t}-\beta \frac{d \rho_{0}^{\prime}}{d t}=0,
\end{aligned}
$$

- Charlier, Die Mechanik des Himmels, vol. 2, 1907, p. 95. 
where $\epsilon=1$ if $a>0$, otherwise $\epsilon=0$. If the left hand members of (11) are zero at $t=t_{0}$, it can easily be shown that as a result of $(11)$ and $\left(1^{\prime}\right)$ their derivatives are zero. Consequently equations (11) are a set of invariant relations, which characterize the straight line solutions.

Suppose

$$
\begin{aligned}
& U_{0}^{\prime}=\frac{1}{\rho_{0}^{\prime}}\left[\frac{m_{0} m_{1}}{(1+a)^{2}}+\frac{m_{0} m_{2}}{(1+\beta)^{2}}\right]=\frac{M_{0}^{\prime} m_{0}}{\rho_{0}^{\prime}}, \\
& U_{1}^{\prime}=\frac{a^{3}}{|a| \rho_{1}^{\prime}}\left[\frac{-m_{1} m_{2}}{(\beta-a)^{2}}+\frac{m_{0} m_{1}}{(1+a)^{2}}\right]=\frac{M_{1}^{\prime} m_{1}}{\rho_{1}^{\prime}}, \\
& U_{2}^{\prime}=\frac{\beta^{2}}{\rho_{2}^{\prime}}\left[\frac{m_{0} m_{2}}{(1+\beta)^{2}}+\frac{m_{1} m_{2}}{(\beta-a)^{2}}\right]=\frac{M_{2}^{\prime} m_{2}}{\rho_{2}^{\prime}}, \\
& F_{i}^{\prime}=\frac{1}{2 m_{i}}\left(R_{i}^{\prime 2}+\frac{\theta_{i}^{\prime 2}}{\rho_{i}^{\prime 2}}\right)-U_{i}^{\prime}
\end{aligned}
$$

By means of equations (9) it can easily be shown that

$$
M_{1}{ }^{\prime}=|a|^{3} M_{0}{ }^{\prime}, \quad M_{2}{ }^{\prime}=\beta^{3} M_{0}{ }^{\prime} .
$$

If equations (11) are satisfied, equations $\left(1^{\prime}\right)$ become

$$
\begin{aligned}
& \frac{d \rho_{i}^{\prime}}{d t}=\frac{\partial F_{i}^{\prime}}{\partial R_{i}^{\prime}}, \quad \frac{d \theta_{i}^{\prime}}{d t}=\frac{\partial F_{i}^{\prime}}{\partial \theta_{i}^{\prime}}, \\
& \frac{d R_{i}{ }^{\prime}}{d t}=-\frac{\partial F_{i}^{\prime}}{\partial \rho_{i}{ }^{\prime}} \quad \frac{d \theta_{i}{ }^{\prime}}{d t}=-\frac{\partial F_{i}{ }^{\prime}}{\partial \theta_{i}{ }^{\prime}} \quad(i=0,1,2),
\end{aligned}
$$

as is easily verified ; from these equations it follows that each mass moves in an ellipse having the center of gravity at one of the foci.

If polar coördinates corresponding to the variables (3) are employed, with the relations

$$
\bar{x}_{3}=\bar{x}_{6}=\bar{y}_{3}=\bar{y}_{6}=0
$$

which correspond to (6), these may be chosen such that $\rho_{1}=r_{2}$, and

$$
\begin{array}{ll}
\bar{x}_{1}=\rho_{1} \cos \varphi_{1}, & \bar{x}_{4}=\rho_{2} \cos \varphi_{2}, \\
\bar{x}_{2}=\rho_{1} \sin \varphi_{1}, & \bar{x}_{5}=\rho_{2} \sin \varphi_{2} .
\end{array}
$$

Suppose

$$
\begin{gathered}
\bar{m}_{1}=\frac{1}{h}, \quad \bar{m}_{2}=\frac{1}{g}, \\
M_{1}=(1+a)^{3} M_{0}^{\prime}, \quad M_{2}=[\beta-a+\mu(1+a)]^{2} M_{0}^{\prime}, \\
U_{1}=\frac{\bar{m}_{1} M_{1}}{\rho_{1}}, \quad U_{2}=\frac{\bar{m}_{2} M_{2}}{\rho_{2}},
\end{gathered}
$$


and

$$
\bar{F}_{1}=\frac{1}{2 \bar{m}_{1}}\left(R_{1}^{2}+\frac{G_{1}^{2}}{\rho_{1}^{2}}\right)-U_{1}, \quad \bar{F}_{2}=\frac{1}{2 \bar{m}_{2}}\left(R_{2}^{2}+\frac{G_{2}^{2}}{\rho_{2}^{2}}\right)-U_{2} .
$$

In terms of the variables (13) equations (4) become

$$
\begin{aligned}
\frac{\bar{x}_{3}=\bar{x}_{6}=\bar{y}_{3}=\bar{y}_{6}=0,}{d \rho_{k}} & =\frac{\partial F}{\partial R_{k}}, \quad \frac{d \varphi_{k}}{d t}=\frac{\partial F}{\partial G_{k}}, \\
\frac{d R_{k}}{d t} & =-\frac{\partial F}{\partial \rho_{k}}, \quad \frac{d G_{k}}{d t}=-\frac{\partial F}{\partial \varphi_{k}} \quad(k=1,2), \\
F & =\frac{1}{2} \sum_{k=1}^{2} \frac{1}{\bar{m}_{k}}\left(R_{k}^{2}+\frac{G_{k}^{2}}{\rho_{k}^{2}}\right)-U .
\end{aligned}
$$

Consider the relations

(a) $\varphi_{2}-\varphi_{1}=0$,

$$
\rho_{2}-(\mu+\nu) \rho_{1}=0 \text {, }
$$

(b) $\frac{d \varphi_{2}}{d t}-\frac{d \varphi_{1}}{d t}=0, \quad \frac{d \rho_{2}}{d t}-(\mu+\nu) \frac{d \rho_{1}}{d t}=0$,

(c) $\quad G_{2}-\frac{\bar{m}_{2}}{\bar{m}_{1}}(\mu+\nu)^{2} G_{1}=0, \quad R_{2}-\frac{\bar{m}_{2}}{\bar{m}_{1}}(\mu+\nu) R_{1}=0$.

As a consequence of equations (16) equations (b) are equivalent to (c) and it can easily be shown that equations (11') follow from (11).

It will be established, independently of $(11)$, that equations $\left(11^{\prime} ; a, c\right)$ form a set of invariant relations; as a consequence of the principle stated by Poincaré $(\$ 1)$ it is only necessary to show that equations $(a)$, (c) with (16) imply that the derivatives of the left members of these equations are zero. Since equations $(c)$ and (16) imply (b), it only remains to show that the derivatives of the left members of $(c)$ are zero, as a consequence of (a), (c), and (16). From (5),

$$
\begin{aligned}
& r_{0}^{2}=\rho_{2}^{2}+\mu^{2} \rho_{1}^{2}-2 \mu \rho_{1} \rho_{2} \cos \left(\varphi_{2}-\varphi_{1}\right), \\
& r_{1}^{2}=\rho_{2}^{2}+\lambda^{2} \rho_{1}^{2}+2 \lambda \rho_{1} \rho_{2} \cos \left(\varphi_{2}-\varphi_{1}\right) .
\end{aligned}
$$

Consequently if $\varphi_{1}=\varphi_{2}$,

$$
\frac{\partial U}{\partial \varphi_{1}}=\frac{\partial U}{\partial \varphi_{2}}=0, \quad \frac{d G_{1}}{d t}=\frac{d G_{2}}{d t}=0
$$


It remains to show that

or from (16), that

$$
\frac{d R_{2}}{d t}=\frac{\bar{m}_{2}}{\bar{m}_{1}}(\mu+\nu) \frac{d R_{1}}{d t},
$$

$$
\frac{G_{2}^{2}}{\bar{m}_{2 \rho_{2}}}+\frac{\partial U}{\partial \rho_{2}}=\frac{\bar{m}_{2}}{\bar{m}_{1}}(\mu+\nu)\left[\frac{G_{1}^{2}}{\bar{m}_{1} \rho_{1}^{\circ}}+\frac{\partial U}{\partial \rho_{1}}\right] .
$$

But from (14), (15), (16) it is easily seen that when equations $\left(11^{\prime} ; a, c\right)$ are satisfied,

$$
\frac{\partial U}{\partial_{\rho_{2}}}=\frac{\partial U_{2}}{\partial_{\rho_{2}}}, \quad \frac{\partial U}{\partial_{\rho_{1}}}=\frac{\partial U_{1}}{\partial_{\rho_{1}}},
$$

and from (14), $\left(11^{\prime}, a\right)$,

from $\left(11^{\prime} ; a, c\right)$

$$
\frac{M_{2}}{\rho_{2}^{2}}=(\mu+\nu) \frac{M_{1}}{\rho_{1}^{2}}
$$

$$
\frac{G_{2}^{2}}{\bar{m}_{2 \rho_{2}^{3}}}=\frac{\bar{m}_{2}}{\bar{m}_{1}}(\mu+\nu) \frac{G_{1}^{2}}{\bar{m}_{1 \rho_{1}^{3}}}
$$

The equality to be established follows immediately, and the relations $\left(11^{\prime}\right)$ are therefore a set of invariant relations, which define the straight line solutions in terms of the variables (13) and the corresponding quantities $R_{i}, G_{i}$. When these relations are satisfied it follows from (15), (16), (17) that the equations of motion are

$$
\begin{aligned}
& \bar{x}_{3}=\bar{x}_{6}=\bar{y}_{3}=\bar{y}_{6}=0, \\
& \frac{d \rho_{k}}{d t}=\frac{\partial \bar{F}_{k}}{\partial R_{k}}, \quad \frac{d \varphi_{k}}{d t}=\frac{\partial \bar{F}_{k}}{\partial G_{k}}, \\
& \frac{d R_{k}}{d t}=-\frac{\partial \bar{F}_{k}}{\partial \rho_{k}}, \quad \frac{d G_{k}}{d t}=-\frac{\partial \bar{F}_{k}}{\partial \varphi_{k}} \quad(k=1,2) .
\end{aligned}
$$

The equations of motion can be simplified by the repeated application of a transformation

$$
\begin{array}{ll}
x_{1}^{\prime}=x_{1}+c x_{2}, & x_{2}^{\prime}=x_{2}, \\
y_{1}^{\prime}=y_{1}, & y_{2}^{\prime}=y_{2}-c y_{1} .
\end{array}
$$

This transformation is such that

$$
x_{1}^{\prime} d y_{1}^{\prime}+x_{2}^{\prime} d y_{2}^{\prime}=x_{1} d y_{1}+x_{2} d y_{2}
$$


if the constant $c$ is supposed given, and hence if $x_{1}, y_{1}, x_{2}, y_{2}$ satisfy equations

$$
\begin{aligned}
\frac{d x_{1}}{d t}=\frac{\partial H}{\partial y_{1}}, & \frac{d x_{2}}{d t}=\frac{\partial H}{\partial y_{2}}, \\
\frac{d y_{1}}{d t}=-\frac{\partial H}{\partial x_{1}}, & \frac{d y_{2}}{d t}=-\frac{\partial B}{\partial x_{2}},
\end{aligned}
$$

the equations will be canonical in terms of the new variables.

Consequently the canonical form of equations (16) is retained by the transformation

$$
\begin{aligned}
\varphi_{1}{ }^{\prime} & =\varphi_{1}, & \varphi & =\varphi_{2}-\varphi_{1}, \\
G_{1}{ }^{\prime} & =G_{1}+G_{2}, & G & =G_{2} .
\end{aligned}
$$

Since $G_{1}+G_{2}=C$, it follows that $\partial F / \partial \varphi_{1}^{\prime}=0$, as is easily verified.* Instead of the variables

$$
\begin{array}{ccc}
\rho_{1}, & \rho_{2}, & \varphi, \\
R_{1}, & R_{2}, & G,
\end{array}
$$

* The canonical reduction of equations $(4, \S 7)$ to four degrees of freedom given by Poincare (Les Méthodes Nouvelles, vol. 1, Chap. 1) can also be obtained by a repetition of this transformation, from the equations in terms of the elliptic coördinates

$$
\begin{aligned}
& l_{1}, l_{2}, g_{1}, g_{2}, \theta_{1}, \theta_{2}, \\
& L_{1}, L_{2}, G_{1}, G_{2}, \theta_{1}, \theta_{2} \quad\left(\theta_{k}=G_{k} \cos i_{k}\right) .
\end{aligned}
$$

If the motion is referred to the invariable plane, from the area integrals one obtains

$$
\theta_{1}+\theta_{2}=C, G_{1} \sin i_{1}+G_{2} \sin i_{2}=0, \theta_{2}=\theta_{1} \text {. }
$$

Applying the transformation above, we obtain the canonical variables

$$
\begin{aligned}
\theta_{1}{ }^{\prime} & =\theta_{1}, & \theta_{2}{ }^{\prime} & =\theta_{2}-\theta_{1}=0, \\
C & =\theta_{1}+\theta_{2}, & \theta_{2}{ }^{\prime} & =\theta_{2},
\end{aligned}
$$

and since $\partial F / \partial \theta_{1}{ }^{\prime}=\partial F / \partial \Theta_{2}{ }^{\prime}$, the variables $\theta_{1}{ }^{\prime}, \theta_{2}{ }^{\prime}$ can be ignored, and the reduced system of Poincare results. If the further transformation

$$
\begin{array}{ll}
h=g_{1}, & g=g_{2}-g_{1}, \\
H=G_{1}+G_{2}-C, & G=G_{2}
\end{array}
$$

is made, then, from the equations above,

$$
H=\frac{4 G_{2}\left(C-G_{2}+H\right) \sin ^{2}\left(\left(i_{1}-i_{2}\right) / 2\right)}{2 C+H} .
$$

These last canonical variables are such that a motion in the invariable plane is characterized by the single invariant relation $B=0$, if $C \neq 0, G_{2}{ }^{0}\left(C-G_{2}{ }^{0}\right) \neq 0$, and when this relation is satisfied the remaining variables define the motion with three degrees of freedom in the plane, while $h$ is ignorable. For if $H^{0}=0, C \neq 0, G_{2}{ }^{0}\left(C-G_{2}{ }^{0}\right) \neq 0$, then $i_{2}-i_{1}=2 \kappa \pi$, consequently from the area integrals we obtain $\left(G_{1}{ }^{0}+G_{2}{ }^{0}\right) \cos i_{1}=C \neq 0, \quad\left(G_{1}{ }^{0}+G_{2}{ }^{0}\right) \sin i_{1}=0$.

Hence $\sin i_{1}=0$, and the inclinations of the two osculating elliptic orbits are zero at the time $t=t^{0}$; whence they must be zero for all values of $t$, and hence $B=0$ for every value of $t$. Consequently

$$
\frac{d H}{d t}=\frac{\partial F}{\partial h}=0 \text {. }
$$


suppose

$$
\begin{aligned}
& \rho_{1}^{\prime}=\rho_{1}, \quad \rho_{2}^{\prime}=\rho_{2}-(\mu+\nu) \rho_{1}, \quad u_{2}=\varphi, \\
& R_{1}^{\prime}=R_{1}+(\mu+\nu) R_{2}, R_{2}^{\prime}=R_{2}, \quad \nu_{2}=G-\frac{\bar{m}_{2}(\mu+\nu)^{2}}{\bar{m}_{1}+\bar{m}_{2}(\mu+\nu)^{2}} C,
\end{aligned}
$$

and finally

$$
\begin{array}{ll}
\rho_{1}^{\prime \prime}=\rho_{1}{ }^{\prime}+\sigma \rho_{2}{ }^{\prime}, & u_{1}=\rho_{2}^{\prime}, \quad u_{2}, \\
R_{1}{ }^{\prime \prime}=R_{1}{ }^{\prime}, & v_{1}=R_{2}{ }^{\prime}-\sigma R_{1}^{\prime}, \nu_{2},
\end{array}
$$

where

$$
\sigma=\frac{\bar{m}_{2}(\mu+\nu)}{\bar{m}_{1}+\bar{m}_{2}(\mu+\nu)^{2}} .
$$

In terms of the last variables the equations (11') become

$$
u_{1}=v_{1}=u_{2}=v_{2}=0 \text {. }
$$

The original variables are

$$
\begin{array}{ll}
\rho_{1}=\rho_{1}^{\prime \prime}-\sigma u_{1}, & \rho_{2}=(\mu+\nu) \rho_{1}^{\prime \prime}+a u_{1}, \\
R_{1}=a R_{1}^{\prime \prime}-(\mu+\nu) v_{1}, & R_{2}=v_{1}+\sigma R_{1}^{\prime \prime}, a=1-\sigma(\mu+\nu), \\
\varphi=u_{2}, & G=v_{2}+\frac{\bar{m}_{2}(\mu+\nu)^{2} C}{\bar{m}_{1}+\bar{m}_{2}(\mu+\nu)^{2}} .
\end{array}
$$

From (16),

$$
F=\frac{1}{2 \bar{m}_{1}}\left[R_{1}^{2}+\frac{(C-G)^{2}}{\rho_{1}^{2}}\right]+\frac{1}{2 \bar{m}_{2}}\left[R_{2}^{2}+\frac{G^{2}}{\rho_{2}^{2}}\right]-U,
$$

$$
r_{0}^{2}=\rho_{2}^{2}+\mu^{2} \rho_{1}^{2}-2 \mu \rho_{1} \rho_{2} \cos \varphi
$$

$$
r_{1}^{2}=\rho_{2}^{2}+\lambda^{2} \rho_{1}^{2}+2 \lambda \rho_{1} \rho_{2} \cos \varphi
$$

After this change of variables equations (16) become

$$
\begin{array}{lll}
\frac{d \rho_{1}^{\prime \prime}}{d t}=\frac{d F}{\partial R_{1}^{\prime \prime}}, & \frac{d u_{1}}{d t}=\frac{\partial F}{\partial v_{1}}, & \frac{d u_{2}}{d t}=\frac{\partial F}{\partial v_{2}}, \\
\frac{d R_{1}^{\prime \prime}}{d t}=-\frac{\partial F}{\partial \rho_{1}^{\prime \prime}}, & \frac{d v_{1}}{d t}=-\frac{\partial F}{\partial u_{1}}, & \frac{d v_{2}}{d t}=-\frac{d F}{\partial u_{2}} .
\end{array}
$$


8. REDUCTION OF THE EQUATIONS OF MOTION IN THE NEIGHBORHOOD OF THE EQUILATERAL TRIANGLE SOLUTION

The equilateral triangle solutions and the solutions in a certain neighborhood of them can be discussed by methods analogous to those employed in \$7. Only plane movements will be treated here.

Suppose the equations of motion in the form $(16,87)$,

$$
\begin{aligned}
\frac{d \rho_{k}}{d t} & =\frac{\partial F}{\partial R_{k}}, \quad \frac{d \varphi_{k}}{d t}=\frac{\partial F}{\partial G_{k}}, \\
\frac{d R_{k}}{d t} & =-\frac{\partial F}{\partial \rho_{k}}, \quad \frac{d G_{k}}{d t}=-\frac{\partial F}{\partial \varphi_{k}} \quad(k=1,2), \\
F & =\frac{1}{2} \sum_{k=1}^{2} \frac{1}{\bar{m}_{k}}\left(R_{k}^{2}+\frac{G_{k}^{2}}{\rho_{k}^{2}}\right)-U,
\end{aligned}
$$

and consider the relations

$$
\rho_{2}-\gamma \rho_{1}=0, \quad \varphi_{2}-\varphi_{1}-\omega=0,
$$

(b)

(c)

$$
\frac{d \rho_{2}}{d t}-\gamma \frac{d \rho_{1}}{d t}=0, \frac{d \varphi_{2}}{d t}-\frac{d \varphi_{1}}{d t}=0 \text {, }
$$

where

$$
R_{2}-\gamma \frac{\bar{m}_{2}}{\bar{m}_{1}} R_{1}=0, G_{2}-\frac{\bar{m}_{2}}{\bar{m}_{1}} \gamma^{2} G_{1}=0,
$$

$$
\gamma=\frac{\sqrt{m_{0}^{2}+m_{0} m_{1}+m_{1}^{2}}}{m_{0}+m_{1}}, \quad \cos \omega=\frac{m_{0}-m_{1}}{2 \sqrt{m_{0}^{2}+m_{0} m_{1}+m_{1}^{2}}} .
$$

Now suppose

$$
\begin{gathered}
\rho_{1}=r_{2}, \quad \bar{M}_{1}=M=m_{0}+m_{1}+m_{2}, \quad \bar{M}_{2}=\gamma^{3} M, \\
U_{1}=\frac{\bar{m}_{1} \bar{M}_{1}}{\rho_{1}}, \quad U_{2}=\frac{\bar{m}_{2} \bar{M}_{2}}{\rho_{2}} .
\end{gathered}
$$

If equations $(2, a)$ are satisfied, one obtains easily

$$
r_{0}=r_{1}=r_{2}, \quad \bar{U}_{1}+\bar{U}_{2}=U,
$$

$$
\frac{\partial U}{\partial \rho_{1}}=\frac{\partial \bar{U}_{1}}{\partial \rho_{1}}, \quad \frac{\partial U}{\partial \rho_{2}}=\frac{\partial \bar{U}_{2}}{\partial \rho_{2}}, \quad \frac{\partial U}{\partial \varphi_{1}}=\frac{\partial U}{\partial \varphi_{2}}=0 .
$$

To show that equations $(2 ; a, c)$ form a set of invariant relations, we shall show that $(1)$ and $(2 ; a, c)$ imply that the derivatives of the left hand 
members of $(2 ; a, c)$ are zero. Since $(1)$ and $(2, c)$ imply $(2, b)$, and the equations (5) imply the relation

$$
\frac{d G_{1}}{d t}=\frac{d G_{2}}{d t}=0
$$

it only remains to show that

or

$$
-\frac{\partial F}{\partial \rho_{2}}+\gamma \frac{\bar{m}_{2}}{\bar{m}_{1}} \frac{\partial F}{\partial \rho_{1}}=0,
$$

$$
\frac{1}{\bar{m}_{2}} \frac{G_{2}^{2}}{\rho_{2}^{3}}+\frac{\partial \bar{U}_{2}}{\partial \rho_{2}}=\gamma \frac{\bar{m}_{2}}{\bar{m}_{1}}\left[\frac{G_{1}^{2}}{\bar{m}_{1} \rho_{1}^{3}}+\frac{\partial \bar{U}_{1}}{\partial \rho_{1}}\right] .
$$

From $(2 ; a, c)$,

$$
\frac{1}{\bar{m}_{2}}-\frac{G_{2}^{2}}{\rho_{2}^{3}}=\gamma \frac{\bar{m}_{2}}{\bar{m}_{1}} \frac{G_{1}^{2}}{\bar{m}_{1} \rho_{1}^{3}},
$$

and from (4)

$$
\frac{\partial \bar{U}_{2}}{\partial_{\rho_{2}}}=\gamma \frac{\bar{m}_{2}}{\bar{m}_{1}} \frac{\partial \bar{U}_{1}}{\partial_{\rho_{1}}} .
$$

Hence, as a consequence of the principal stated by Poincaré, the relations $(2 ; a, c)$ form a set of invariant relations. When these relations are satisfied, it follows from the discussion just given that if

$$
\begin{aligned}
& F_{1}=\frac{1}{2 \bar{m}_{1}}\left(R_{1}^{2}+\frac{G_{1}^{2}}{\rho_{1}^{2}}\right)-\bar{U}_{1}, \\
& F_{2}=\frac{1}{2 \bar{m}_{2}}\left(R_{2}^{2}+\frac{G_{2}^{2}}{\rho_{2}^{2}}\right)-\bar{U}_{2},
\end{aligned}
$$

the equations (1) become

$$
\frac{d \rho_{1}}{d t}=\frac{\partial F_{i}}{\partial R_{i}}, \quad \frac{d \varphi_{i}}{d t}=\frac{\partial F_{i}}{\partial G_{i}},
$$

$$
\frac{d R_{i}}{d t}=-\frac{\partial F_{i}}{\partial \rho_{i}}, \quad \frac{d G_{i}}{d t}=-\frac{\partial F_{i}}{\partial \varphi_{i}}
$$

From these equations the elliptic form of the relative orbits is evident. As in the preceding paragraph, if the change of variables

$$
\begin{aligned}
\varphi_{1}{ }^{\prime} & =\varphi_{1}, & & \varphi=\varphi_{2}-\varphi_{1}, \\
C & =G_{1}+G_{2}, & G & =G_{2}
\end{aligned}
$$


is made, $C=$ const., and the motion is defined by the canonical variables

$$
\begin{array}{lll}
\rho_{1}, & \rho_{2}, & \varphi, \\
R_{1}, & R_{2}, & G .
\end{array}
$$

Suppose the canonical change of variables made,

$$
\begin{aligned}
& \rho_{1}^{\prime}=\rho_{1}, \\
& \rho_{2}^{\prime}=\rho_{2}-\gamma \rho_{1}, \quad u_{2}=\varphi-\omega, \\
& R_{1}{ }^{\prime}=R_{1}+\gamma R_{2} \text {, } \\
& R_{2}{ }^{\prime}=R_{2} \text {, } \\
& v_{2}=G-\sigma^{\prime} C \\
& \left(\sigma^{\prime}=\frac{\bar{m}_{2} \gamma^{2}}{\bar{m}_{1}+\bar{m}_{2} \gamma^{2}}\right) \text {, }
\end{aligned}
$$

and finally

$$
\begin{array}{lll}
\rho_{1}{ }^{\prime \prime}=\rho_{1}{ }^{\prime}+m u_{1}, & u_{1}=\rho_{2}-\gamma \rho_{1}, & u_{2}=\varphi-\omega, \\
R_{1}{ }^{\prime \prime}=R_{1}{ }^{\prime}, & v_{1}=R_{2}{ }^{\prime}-m R_{1}{ }^{\prime}, & v_{2}=G-\sigma^{\prime} C, \\
& m=\frac{\bar{m}_{2} \gamma}{\bar{m}_{1}+\gamma^{2} \bar{m}_{2}} .
\end{array}
$$

The relations $(2 ; a, c)$ become

$$
u_{1}=v_{1}=u_{2}=v_{2}=0,
$$

and the equations of motion

$$
\begin{aligned}
\frac{d \rho_{1}{ }^{\prime \prime}}{d t}=\frac{\partial F}{\partial R_{1}{ }^{\prime \prime}}, & \frac{d u_{1}}{d t}=\frac{\partial F}{\partial v_{1}}, & \frac{d u_{2}}{d t}=\frac{\partial F}{\partial v_{2}}, \\
\frac{d R_{1}^{\prime \prime}}{d t}=-\frac{\partial F}{\partial \rho_{1}{ }^{\prime \prime}}, & \frac{d v_{1}}{d t}=-\frac{\partial F}{\partial u_{1}}, & \frac{d v_{2}}{d t}=-\frac{\partial F}{\partial u_{2}} .
\end{aligned}
$$

The original variables are defined by the equations

$$
\begin{array}{lll}
R_{1}=(1-m \gamma) R_{1}{ }^{\prime \prime}-\gamma v_{1}, & \rho_{1}=\rho_{1}{ }^{\prime \prime}-m u_{1}, & \varphi=\omega+u_{2}, \\
R_{2}=m R_{1}^{\prime \prime}+v_{1}, & \rho_{2}=\gamma \rho_{1}^{\prime \prime}+(1-m \gamma) u_{1}, & G=\sigma^{\prime} C+v_{2} .
\end{array}
$$

9. ORBITS IN THE NEIGHBORHOOD OF THE

$$
\text { STRAIGHT LINE SOLUTIONS }
$$

The behavior of the solutions of equations $(21, \S 7)$, in the neighborhood of a straight line solution depends upon the properties of the equations of variation, corresponding to a solution for which $u_{1}=v_{1}=u_{2}=v_{2}=0$.

Since

$$
\begin{aligned}
r_{0}^{2} & =\rho_{2}^{2}+\mu^{2} \rho_{1}^{2}-2 \mu \rho_{1} \rho_{2}+2 \mu \rho_{1} \rho_{2}(1-\cos \varphi) \\
& =\left(\rho_{2}-\mu \rho_{1}\right)^{2}\left[1+\frac{4 \mu \rho_{1} \rho_{2}}{\left(\rho_{2}-\mu \rho_{1}\right)^{2}} \sin ^{2} \frac{\varphi}{2}\right],
\end{aligned}
$$


we obtain

$$
\frac{1}{r_{0}}=\frac{1}{\rho_{2}-\mu \rho_{1}}\left[1-\frac{2 \mu \rho_{1} \rho_{2}}{\left(\rho_{2}-\mu \rho_{1}\right)^{2}} \sin ^{2} \frac{\varphi}{2}+\frac{1.3}{2.4}\left(\frac{4 \mu \rho_{1} \rho_{2}}{\left(\rho_{2}-\mu \rho_{1}\right)^{2}} \sin ^{2} \frac{\varphi}{2}\right)^{2}+. .\right]
$$

and, similarly,

$$
\frac{1}{r_{1}}=\frac{1}{\rho_{2}+\lambda \rho_{1}}-\left[1+\frac{2 \lambda \rho_{1} \rho_{2}}{\left(\rho_{2}+\lambda \rho_{1}\right)^{2}} \sin ^{2} \frac{\varphi}{2}+\frac{1 \cdot 3}{2 \cdot 4}\left(\frac{4 \lambda \rho_{1} \rho_{2}}{\left(\rho_{2}+\lambda \rho_{1}\right)^{2}} \sin ^{2} \frac{\varphi}{2}\right)^{2}+. .\right] .
$$

Consequently

$$
\begin{aligned}
U=\frac{m_{1} m_{2}}{\rho_{2}-\mu \rho_{1}}+\frac{m_{2} m_{0}}{\rho_{2}}+\lambda \rho_{1} & +\frac{m_{0} m_{1}}{\rho_{1}} \\
& +2 \rho_{1} \rho_{2}\left[\frac{\lambda m_{2} m_{0}}{\left(\rho_{2}+\lambda \rho_{1}\right)^{3}}-\frac{\mu m_{1} m_{2}}{\left(\rho_{2}-\mu \rho_{1}\right)^{3}}\right] \sin ^{2} \frac{u_{2}}{2}+U_{3},
\end{aligned}
$$

where $U_{3}$ is a power series in $\sin ^{2}\left(u_{2} / 2\right)$ of at least the second degree in $\sin ^{2}\left(u_{2} / 2\right)$. It follows immediately that

$$
\frac{\partial^{2} F}{\partial u_{2}^{2}}=-\rho_{1} \rho_{2}\left[\frac{\lambda m_{2} m_{0}}{\left(\rho_{2}+\lambda \rho_{1}\right)^{3}}-\frac{\mu m_{1} m_{2}}{\left(\rho_{2}-\mu \rho_{1}\right)^{3}}\right]+u_{2}(\cdots),
$$

and when $u_{1}=v_{1}=u_{2}=v_{2}=0$,

If

$$
\frac{\partial^{2} F}{\partial u_{2} \partial u_{1}}=\frac{\partial^{2} F}{\partial u_{2} \partial v_{1}}=\frac{\partial^{2} F}{\partial u_{2} \partial v_{2}}=0 \text {. }
$$

$$
\begin{gathered}
A_{11}=\frac{\partial^{2} F}{\partial u_{1}^{2}}, \quad A_{22}=\frac{\partial^{2} F}{\partial v_{1}^{2}}, \quad B_{11}=\frac{\partial^{2} F}{\partial u_{2}^{2}} \\
B_{12}=B_{21}=\frac{\partial^{2} F}{\partial u_{1} \partial v_{2}}, \quad B_{22}=\frac{\partial^{2} F}{\partial v_{2}^{2}}
\end{gathered}
$$

all the remaining second order partial derivatives of $F$ are zero under the conditions above; if $e$ is the excentricity of the straight line solutions, $a_{1}$ the semi-major axis of the ellipse whose radius vector is $\rho_{1}$, we obtain

$$
\begin{aligned}
& A_{11}=\frac{3 \bar{m}_{1} a_{1}\left(1-e^{2}\right) \sigma M_{1}}{(\mu+\nu) \rho_{1}^{4}}-\frac{2}{\rho_{1}^{3}}\left[\frac{m_{1} m_{2}(1-\sigma \nu)^{2}}{\nu^{3}}+\frac{m_{2} m_{0}(a-\lambda \sigma)^{2}}{(1+\nu)^{3}}+m_{0} m_{1} \sigma^{2}\right], \\
& A_{22}=\frac{\mu+\nu}{\bar{m}_{1} \sigma}, \quad B_{11}=\frac{(\mu+\nu) m_{0} m_{1} m_{2}}{\left(m_{0}+m_{1}\right) \rho_{1}}\left[\frac{1}{\nu^{3}}-\frac{1}{(1+\nu)^{3}}\right], \\
& B_{12}=\frac{-2 \sqrt{M_{1} a_{1}\left(1-e^{2}\right)}}{(\mu+\nu) \rho_{1}^{3}}, \quad B_{22}=\frac{1}{\bar{m}_{1} \sigma(\mu+\nu) \rho_{1}^{2}} .
\end{aligned}
$$


Since $u_{i}=v_{i}=0$ form a set of invariant relations, the equations of variation are necessarily in the form of $\S 2$; consequently, if $\rho_{1}^{\prime \prime}=\rho_{1}^{0}+\xi, R_{1}^{\prime \prime}=$ $R_{1}^{0}+\eta$

$$
\begin{aligned}
& \frac{d \xi}{d t}=\frac{\partial^{2} F}{\partial R_{1}{ }^{\prime \prime 2}} \eta, \quad \frac{d \eta}{d t}=-\frac{\partial^{2} F}{\partial \rho_{1}{ }^{\prime 2}} \xi, \\
& \frac{d \bar{u}_{1}}{d t}=\quad A_{22} \bar{v}_{1} \text {, } \\
& \frac{d \bar{u}_{2}}{d t}=B_{12} \bar{u}_{1} \quad+B_{22} \bar{\nu}_{2}, \\
& \frac{d \bar{v}_{1}}{d t}=-A_{11} \bar{u}_{1} \quad-B_{12} \bar{v}_{2}, \\
& \frac{d \bar{v}_{2}}{d t}=\quad-B_{11} \bar{u}_{2} .
\end{aligned}
$$

If $e=0$ for the straight line solutions, the characteristic exponents different from zero are obtained from the equation

$$
\Delta(S)=\left|\begin{array}{cccc}
-s & 0 & A_{22} & 0 \\
B_{12} & -s & 0 & B_{22} \\
-A_{11} & 0 & -s & -B_{12} \\
0 & -B_{11} & 0 & -s
\end{array}\right|=0,
$$

or

$$
s^{4}+\left[A_{11} A_{22}+B_{11} B_{22}\right] s^{2}+B_{11} A_{22}\left[A_{11} B_{22}-B_{12}^{2}\right]=0 .
$$

Now $B_{11} A_{22}>0, B_{22}>0$, and if $A_{11}^{\prime}$ is the first term of the expression for $A_{11}$, it is found immediately that

$$
A_{11}^{\prime} B_{22}-B_{12}^{2}=\frac{-M_{1}}{(\mu+\nu)^{2} a_{1}^{5}} ;
$$

consequently the product of the two roots $s_{1}^{2}, s_{2}^{2}$ of the equation above is negative. Hence two of the characteristic exponents are real, and two are pure imaginaries. It is easy to find particular cases in which none of these exponents are multiples of $n i$ if $T=2 \pi / n$ is the period of the generating solution.

10. ORBITS IN THE NEIGHBORHOOD OF THE EQUILATERAL TRIANGLE SOLUTIONS

As in the preceding paragraph, we shall set up the equations of variation for the equilateral triangle solution considered. 
From the relations

$$
\begin{aligned}
& r_{0}^{2}=\rho_{2}^{2}+\mu^{2} \rho_{1}^{2}-2 \mu \rho_{1} \rho_{2} \cos \left(\omega+u_{2}\right), \\
& r_{1}^{2}=\rho_{2}^{2}+\lambda^{2} \rho_{1}^{2}+2 \lambda \rho_{1} \rho_{2} \cos \left(\omega+u_{2}\right), \\
& F=\frac{1}{2 \bar{m}_{1}}\left(R_{1}^{2}+\frac{(C-G)^{2}}{\rho_{1}^{2}}\right)+\frac{1}{2 \bar{m}_{2}}\left(R_{2}^{2}+\frac{G^{2}}{\rho_{2}^{2}}\right)-U, \\
& U=\frac{m_{1} m_{2}}{r_{0}}+\frac{m_{2} m_{0}}{r_{1}}+\frac{m_{0} m_{1}}{\rho_{1}}
\end{aligned}
$$

we obtain easily the expansion of $F$ in powers of $u_{i}, v_{i}$. From the fact that the relations $u_{i}=v_{i}=0$ form a set of invariant relations it follows immediately that

$$
\left[\frac{\partial F}{\partial u_{i}}\right]_{0}=\left[\frac{\partial F}{\partial v_{i}}\right]_{0}=0
$$

if these derivatives are computed for an equilateral triangle solution. If

$$
\begin{array}{lll}
A_{11}=\frac{\partial^{2} F}{\partial u_{1}^{2}}, & A_{12}=\frac{\partial^{2} F}{\partial u_{1} \partial u_{2}}, & B_{12}=\frac{\partial^{2} F}{\partial u_{1} \partial v_{2}}, \\
A_{22}=\frac{\partial^{2} F}{\partial u_{2}^{2}}, & B_{11}=\frac{\partial^{2} F}{\partial v_{1}^{2}}, & B_{22}=\frac{\partial^{2} F}{\partial v_{2}^{2}},
\end{array}
$$

the values of $R_{1}^{\prime \prime}, \rho_{1}^{\prime \prime}$ for an equilateral triangle solution being substituted in these derivatives, we obtain

$$
\begin{aligned}
A_{11}= & \frac{3 G_{1}^{2} m^{2}}{m_{1} \rho_{1}^{4}}+\frac{3 G_{2}^{2}(1-m \gamma)^{2}}{\bar{m}_{2} \rho_{2}^{4}}+\frac{m_{1} m_{2}}{\rho_{1}^{3}}\left[m^{2} \mu^{2}+(1-m \gamma)^{2}\right] \\
& +\frac{m_{2} m_{0}}{\rho_{1}^{3}}\left[m^{2} \lambda^{2}+(1-m \gamma)^{2}\right]-\frac{3 m_{1} m_{2}}{\rho_{1}^{5}}[m \mu R-(1-m \gamma) \bar{U}]^{2} \\
& -\frac{3 m_{2} m_{0}}{\rho_{1}^{5}}[m \lambda S-(1-m \gamma) V]^{2}-\frac{2 m^{2} m_{0} m_{1}}{\rho_{1}^{3}}, \\
A_{12}= & \frac{3 m_{0} m_{1} m_{2} \gamma \sin \omega \cos \omega}{\left(m_{0}+m_{1}\right) \rho_{1}^{2}}, \quad B_{12}=\frac{-2 m_{1} G_{1}}{\bar{m}_{1} \rho_{1}^{3}}-\frac{2(1-m \gamma) G_{2}}{\bar{m}_{2} \rho_{2}^{3}}, \\
A_{22}= & \frac{-3 m_{0} m_{1} m_{2} \gamma^{2} \sin ^{2} \omega}{\left(m_{0}+m_{1}\right) \rho_{1}},
\end{aligned}
$$




$$
B_{11}=\frac{\gamma^{2}}{\bar{m}_{1}}+\frac{1}{\bar{m}_{2}}, \quad B_{22}=\frac{B_{11}}{\rho_{1}^{2} \gamma^{2}},
$$

where

$$
\begin{array}{ll}
\bar{U}=\rho_{2}-\mu \rho_{1} \cos \omega, & V=\rho_{2}+\lambda \rho_{1} \cos \omega, \\
R=\mu \rho_{1}-\rho_{2} \cos \omega, & S=\lambda \rho_{1}+\rho_{2} \cos \omega .
\end{array}
$$

It is seen that all the remaining partial derivatives of the second order vanish for an equilateral triangle solution. The equations of variation for these solutions become, if

$$
\begin{array}{ll}
\rho_{1}^{\prime \prime}=\rho_{1}^{0}+\xi, & R_{1}^{\prime \prime}=R_{1}^{0}+\eta, \\
\frac{d \bar{\xi}}{d t}=\frac{\partial^{2} F}{\partial R_{1}{ }^{\prime 2}} \bar{\eta}, & \frac{d \eta}{d t}=-\frac{\partial^{2} F}{\partial \rho_{1}{ }^{\prime \prime 2}} \bar{\xi}, \\
\frac{d \bar{u}_{1}}{d t}= & B_{11} \bar{v}_{1}, \\
\frac{d \bar{u}_{2}}{d t}=B_{11} \bar{u}_{1} & +B_{22} \bar{v}_{2}, \\
\frac{d \bar{v}_{1}}{d t}=-A_{11} \bar{u}_{1}-A_{12} \bar{u}_{2} & -B_{12} \bar{v}_{2}, \\
\frac{d \bar{v}_{2}}{d t}=-A_{12} \bar{u}_{1}-A_{22} \bar{u}_{2} . &
\end{array}
$$

If the eccentricity of the generating orbit is zero, the coefficients $A_{i j}$, $B_{i j}$ are constant, and the characteristic exponents of the given orbit satisfy the equation

$$
\Delta^{\prime}(s)=\left|\begin{array}{cccc}
-s & 0 & B_{11} & 0 \\
B_{12} & -s & 0 & B_{22} \\
-A_{11} & -A_{12} & -s & -B_{12} \\
-A_{12} & -A_{22} & 0 & -s
\end{array}\right|=0 \text {, }
$$

or

$$
s^{4}+\left[A_{11} B_{11}+A_{22} B_{22}\right] s^{2}+A_{22} B_{11}\left[A_{11} B_{22}-B_{12}^{2}\right]-B_{11} B_{22} A_{12}^{2}=0
$$

This equation becomes, after some reductions, *

$$
s^{4}+\frac{m_{0}+m_{2}-m_{2}}{a^{3}} s^{2}+\frac{27}{4 a^{6}}\left(m_{0} m_{1}+m_{1} m_{2}+m_{2} m_{0}\right)=0 .
$$

* For a different deduction of a similar equation, see D. Buchanan, these Transactions, loc. cit., p. 415 , equation (11). 
11. ORBITS ASYMPTOTIC TO THE STRAIGHT LINE AND EQUILATERAL TRIANGLE SOLUTIONS, RESPECTIVELY

If in equations $\left(1^{\prime \prime}, \S 8\right)$ we put $\rho_{1}{ }^{\prime \prime}=\rho_{1}^{0}+\xi, R_{1}{ }^{\prime \prime}=R_{1}^{0}+\eta$, and expand $F$ in powers of $\xi$ and $\eta$, where $\rho_{1}^{0}, R_{1}^{0}$ satisfy the equations of the straight line solution, $F$ is of the second degree with respect to $\eta$, but a power series in $\xi$. If the equations of variation for $\bar{\xi}, \bar{\eta}$ are integrated, the solutions are periodic of period $2 \pi / n$, and accordingly the characteristic exponents are $\pm n i$.

From the theory of linear equations with periodic coefficients it follows that by means of one linear transformation with periodic coefficients the equations in $\bar{\xi}, \bar{\eta}$ can be reduced to a system with constant coefficients, and the equations in $\bar{u}_{i}, \bar{v}_{i}$ can be similarly reduced by means of a linear transformation with coefficients of period $2 \pi / n$ in $t$. These equations can in fact be given the form

(1)

$$
\frac{d \bar{\xi}}{d t}=n i \bar{\xi}^{\prime}, \quad \frac{d \eta^{\prime}}{d t}=-n i \bar{\eta}^{\prime},
$$

$$
\begin{array}{ll}
\frac{d \bar{u}_{1}^{\prime}}{d t}=s_{1} \bar{u}_{1}^{\prime}, & d \bar{u}_{2}^{\prime}=s_{2} \bar{u}_{2}^{\prime}, \\
\frac{d \bar{v}_{1}^{\prime}}{d t}=s_{3} \bar{v}_{1}^{\prime}, & \frac{d \bar{v}_{2}^{\prime}}{d t}=s_{4} \bar{v}_{2}^{\prime} .
\end{array}
$$

If the variables $\xi, \eta, u_{i}, v_{i}$ are subjected to the same linear transformation with periodic coefficients, the equations of motion $(21, \S 7)$ become, after this transformation,

(2)

$$
\begin{aligned}
& \frac{d \xi^{\prime}}{d t}=n i \xi+\varphi_{2}^{\prime}\left(\xi, \eta, u_{1}^{\prime}, u_{2}^{\prime}, v_{1}^{\prime}, v_{2}^{\prime}, t\right), \\
& \frac{d \eta^{\prime}}{d t}=-n i \eta^{\prime}+\varphi_{2}^{\prime \prime}, \\
& \frac{d u_{1}^{\prime}}{d t}=s_{1} u_{1}^{\prime}+\varphi_{2}^{\prime \prime \prime}, \\
& \frac{d u_{2}^{\prime}}{d t}=s_{2} u_{2}^{\prime}+\varphi_{2}^{(1)}, \\
& \frac{d v_{1}^{\prime}}{d t}=s_{3} v_{1}^{\prime}+\varphi_{2}^{(5)}, \\
& \frac{d v_{2}^{\prime}}{d t}=s_{1} v_{2}^{\prime}+\varphi_{2}^{(0)},
\end{aligned}
$$


where $\varphi_{2}{ }^{\prime}, \cdots, \varphi_{2}^{(6)}$ are of at least the second degree with respect to the variables $\xi^{\prime}, \eta^{\prime}, u_{i}^{\prime}, v_{i}^{\prime}$ and periodic of period $T=2 \pi / n$ with respect to $t$. We can at once apply the method of Picard to determine solutions of (2) developed in powers of $e^{s_{1} t}$ if $s_{1}$ has its real part negative, and it has been seen that there is just one characteristic exponent having this property, the others being pure imaginaries or positive. Hence no sum of the form

$$
p_{1} s_{1}+\gamma^{\prime} i-s_{h}
$$$$
(h=1,2,3)
$$

can vanish, if $p_{1} \geqq 2$. The variable $\theta$ of Picard* is here $e^{t}$; the application of Picard's method gives one solution developed in powers of $e^{\text {st }}$ which is such that $\xi^{\prime}, \eta^{\prime}, u_{i}{ }^{\prime}, v_{i}{ }^{\prime}$ approach zero as $t \rightarrow \infty$; by choosing the other real exponent one obtains in precisely the same manner a solution approaching the straight line solution as $t \rightarrow-\infty$.

The equations of motion for the equilateral triangle solution can be transformed into the form (2) in precisely the same manner, and the method of Picard can again be applied.

In this case two exponents $s_{1}, s_{2}$ have their real parts negative, hence, for certain ranges of the masses and $e$, solutions can be obtained developed in powers of $e^{s t}, e^{s t}$ which approach the equilateral triangle solutions as $t \rightarrow \infty$. In the same manner another family of solutions can be obtained, which approach the equilateral triangle solutions as $t \rightarrow-\infty$; the variables are here developed in powers of $e^{s t}, e^{s t}$, the coefficients being again periodic in $t$ of period $T=2 \pi / n$.

Solutions asymptotic to the straight line solutions for which the excentricity is zero have been discussed by D. Buchanan $\dagger$ for arbitrary masses ; it follows from the results of $\S 9$ that of the four cases mentioned by him only Case $I$ is possible.

While the series obtained in this manner are not real, the arbitrary constants can be chosen as conjugate imaginaries, and the periodic functions which enter into the transformations can be chosen in conjugate pairs in such a manner that the original variables are real functions of $t$. The calculation of these series is outlined very completely in the two papers of D. Buchanan, following another method, if $e=0$ for the generating solution; with the variables above and the method of Picard the corresponding asymptotic solutions can be obtained for $e$ arbitrary within certain limits.

* Traité d' Analyse, vol. 3, Chap. 8 (2d edition).

† Rendiconti del Circolo Matematico Palermo, vol. 45 (1921), pp. 1-25.

Dalhouste University, Halifax, Nova Scotia 